Article

\title{
An Enhanced LoRaWAN Security Protocol for Privacy Preservation in IoT with a Case Study on a Smart Factory-Enabled Parking System
}

\author{
Ilsun You ${ }^{\mathbb{D}}$, Soonhyun Kwon, Gaurav Choudhary, Vishal Sharma * ${ }^{\mathbb{D}}$ and Jung Taek Seo \\ Department of Information Security Engineering, Soonchunhyang University, Asan 31538, Korea; \\ ilsunu@gmail.com (I.Y.); tnsgus08@gmail.com (S.K.); gauravchoudhary7777@gmail.com (G.C.); \\ seojt@sch.ac.kr (J.T.S.) \\ * Correspondence: vishal_sharma2012@hotmail.com
}

Received: 10 May 2018; Accepted: 5 June 2018; Published: 8 June 2018

\begin{abstract}
The Internet of Things (IoT) utilizes algorithms to facilitate intelligent applications across cities in the form of smart-urban projects. As the majority of devices in IoT are battery operated, their applications should be facilitated with a low-power communication setup. Such facility is possible through the Low-Power Wide-Area Network (LPWAN), but at a constrained bit rate. For long-range communication over LPWAN, several approaches and protocols are adopted. One such protocol is the Long-Range Wide Area Network (LoRaWAN), which is a media access layer protocol for long-range communication between the devices and the application servers via LPWAN gateways. However, LoRaWAN comes with fewer security features as a much-secured protocol consumes more battery because of the exorbitant computational overheads. The standard protocol fails to support end-to-end security and perfect forward secrecy while being vulnerable to the replay attack that makes LoRaWAN limited in supporting applications where security (especially end-to-end security) is important. Motivated by this, an enhanced LoRaWAN security protocol is proposed, which not only provides the basic functions of connectivity between the application server and the end device, but additionally averts these listed security issues. The proposed protocol is developed with two options, the Default Option (DO) and the Security-Enhanced Option (SEO). The protocol is validated through Burrows-Abadi-Needham (BAN) logic and the Automated Validation of Internet Security Protocols and Applications (AVISPA) tool. The proposed protocol is also analyzed for overheads through system-based and low-power device-based evaluations. Further, a case study on a smart factory-enabled parking system is considered for its practical application. The results, in terms of network latency with reliability fitting and signaling overheads, show paramount improvements and better performance for the proposed protocol compared with the two handshake options, Pre-Shared Key (PSK) and Elliptic Curve Cryptography (ECC), of Datagram Transport Layer Security (DTLS).
\end{abstract}

Keywords: LoRaWAN; privacy; IoT; smart parking; security; protocol

\section{Introduction}

The Internet of Things (IoT) with its principles of "connectivity to all" and "connectivity with all" has become a crucial part of the telecommunication system [1-3]. IoT facilities many social issue-solving applications such as smart cities, intelligent transportation, urban surveillance, day-work management and smart-farming [4-8]. With the increasing popularity of IoT, the number of connected devices is bound to increase exponentially. The IoT network can be scaled for improved performance by the use of software-defined and application-aware networking $[9,10]$. The software technologies can be 
modeled to enhance the privacy rules and help to enhance the authentication procedures [11-13]. With effective strategies for collaborative applications, IoT can be used for long, as well as short distance communications [14-17].

In the past, IoT was emphasized to incorporate many short-range communications technologies [18-22], which sometimes present an obstacle for efficient working of devices. As an alternative, Low Power Wide Area Network (LPWAN) technologies are adopted with the property of long-range and low-power computation, but for low-bit rate requirements [23-26]. However, with the short-distance communications, incorporation of IoT into cyber-physical systems and cloud architectures is always a challenge because of security concerns and a lack of privacy preservation over limited range spectrums [27-29]. These security issues worsen when the underlying architecture is used for real-time applications with the involvement of crucial user data [30-34].

Recently, the Long-Range Wide Area Network (LoRaWAN) has become one of the most significant technologies for LPWAN due to its property of long-range communication with energy-efficient computations [35]. These features help to maintain the trade-off between the network latency and the battery lifetime. LoRaWAN also effectively complies with specific features of IoT through the dedicated physical and Medium Access Control (MAC) layer.

The innovative features of the LoRaWAN network are the reasons for compatibility with many low-power applications involving IoT, smart cities, and industrial applications [36,37]. Its advantages are formulated in terms of bandwidth, battery life, range, latency and throughput. Recently, LoRaWAN has been influenced by the standard properties and adopted as a standard mechanism for resource-constrained networks [38].

With the advantages and an enhanced scope of improvements, the LoRaWAN network has already become an emerging area of research. In spite of being well designed, the LoRaWAN network faces several security vulnerabilities, which have been pointed out by many researchers [39-42]. In more detail, it fulfills the basic security properties, but suffers from the following vulnerabilities. First, its join procedure causes a vulnerability, which leads to exploitation by replay attacks. Second, the protocol cannot provide end-to-end security because the application session key between each device and its application server is established with the help of the core network. In other words, the traffic between the two parties can be easily known by the LoRaWAN network server. Third, the network and application session keys, which are established based on a long-term shared key, cannot provide perfect forward secrecy. Considering that every device can be easily broken and compromised, their long-term key can also be exposed, thereby causing the past session keys and their encrypted data to be recovered. It is obvious that the security flaws mentioned above present an obstacle to the successful settlement of the LoRaWAN network.

In order to address these security flaws, several types of research have been conducted [41-44]. However, they are lacking in terms of implementation, while maintaining the standard spectrum. Most of them need changes in the existing standard protocol. Therefore, a more secure and effective low-power consumption scheme is required, which is acceptable under the benchmarks of the existing standard. On the other hand, in the LoRaWAN network, it can be considered to apply Datagram Transport Layer Security (DTLS) [45] to provide the end-to-end security between each device and its application server. However, the DTLS handshake procedure results in excessive message signaling and computation overheads, which are not clearly suited for the LoRaWAN network. As an alternative, we can design a lightweight version for the authentication and key exchange between the two parties, which is the strong motivation of this paper.

The goal of this paper is to bring out a comprehensive analysis of the LoRaWAN's security scheme and the existing solutions for its limitations, as well as provide an effective remedy for their problems. Along with such analyses, a secure scheme is proposed to focus on addressing the replay attacks and achieving both the perfect forward secrecy and the end-to-end security between each device and its application server. Note that the proposed protocol can be divided into two parts where the first one is the standard join procedure and the second one is the key exchange protocol. After the 
proposed protocol (i.e., the strong master session key is established between a device and its application server), the two parties can run the DTLS record protocol based on the established key. The proposed protocol supports the majority of the security properties including mutual authentication, secret key exchange, perfect forward secrecy, end-to-end security and defense against the replay attack to support the security-sensitive applications the should keep the end-to-end security. The proposed protocol is formally analyzed for its security through Burrows-Abadi-Needham (BAN) logic [46] and the Automated Validation of Internet Security Protocols and Applications (AVSIPA) tool [47]. Further, the performance analysis is presented in comparison with the DTLS's two handshake options, Pre-Shared Key (PSK) and Elliptic Curve Cryptography (ECC), along with a case study on a smart factory-enabled parking system $[45,48]$. In the case study, the proposed protocol is analyzed for its performance by securing communication between the end devices (sensors) at the parking lot and the application server, which is hosted by the smart factory, as shown in Figure 1. The results are analyzed for network latency with reliability fitting and signaling overheads for the proposed protocol.

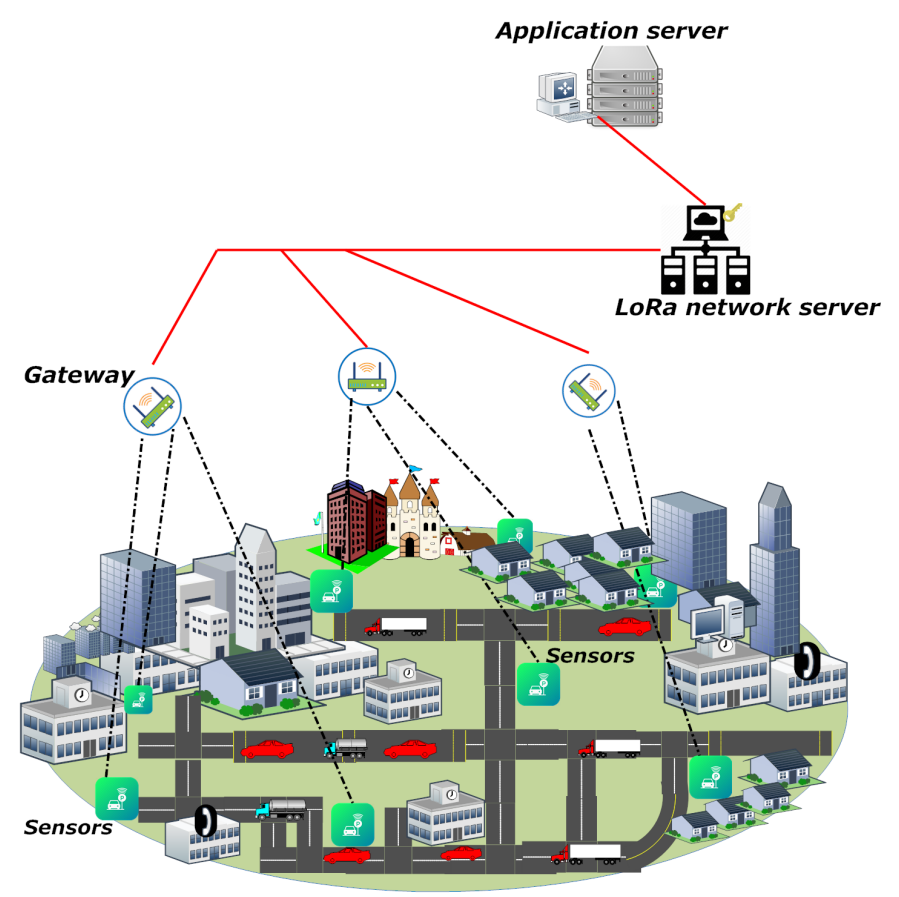

Figure 1. An exemplary illustration of the LoRaWAN-enabled network architecture.

The rest of this paper is structured as follows: Section 2 provides details of LoRaWAN and its functionalities. Section 3 presents insight into the related works on LoRaWAN security. Section 4 gives the details on the proposed protocol, its functioning and policies. Analyses through BAN logic and the AVISPA tool are presented in Section 5. Performance evaluations with the smart parking case study are presented in Section 6. Finally, Section 7 concludes the paper along with future directions and remarks.

\section{Background}

This section presents details on LoRaWAN, its architecture, key exchange policies and procedures $[49,50]$. The basic notations used to describe the LoRaWAN join procedure and the proposed protocol are provided in Table 1.

\subsection{LoRaWAN Network Architecture}

LoRaWAN is designed to be used for battery drain applications where low power consumption with long-range communication is a primary objective. In the LoRaWAN specification v1.02 [51], network range is defined to be $5-15 \mathrm{~km}$, data rates are between $0.3 \mathrm{kbps}$ and $50 \mathrm{kbps}$ and the network is 
operated over the 868-MHz and 900-MHz ISM bands. LoRaWAN, based on the star topology, has grown as one of the most popular technologies for IoT. Its architecture aims to provide interoperability among IoT devices irrespective of their characteristics.

Table 1. Notations table.

\begin{tabular}{|c|c|}
\hline Symbol & Description \\
\hline Join_request & Join request to attach the end device to the LoRa network \\
\hline AppEUI & Application identifier \\
\hline DevEUI & Device identifier \\
\hline DevNonce & Nonce value randomly generated by the device \\
\hline DevNonce $_{i}$ & The $i$-th nonce value computed by the device \\
\hline$A E S 128-C M A C(K, M)$ & AES 128 cipher-based MAC function with the secret key $K$ and the message $M$ \\
\hline MIC & Message integrity code \\
\hline$M I C_{i}$ & The $i$-th message integrity code except for the digital signatures $M I C_{3 a}$ and $M I C_{4}$ \\
\hline NwkSKey & Network session key \\
\hline AppSKey & Application session key \\
\hline AppKey & The long-term key shard between a device and a network server \\
\hline AppNonce & Nonce value randomly generated by the network server \\
\hline AppNonce $_{i}$ & The $i$-th nonce value computed by the network server \\
\hline NetID & Network identifier \\
\hline DevAddr & End device address \\
\hline RxDelay & Delay between RX and TX \\
\hline CFList & Optional list for channel frequencies \\
\hline $\operatorname{sh} a_{1}(M)$ & SHA 1 hash function, which takes an input $M$ and produces a 160-bit \\
\hline App_Auth_Req & Application authentication request message \\
\hline App_Auth_Res & Application authentication response message \\
\hline $\mathrm{Seq}_{i}$ & The $i$-th sequence number \\
\hline$S K$ & The session key between a device and its application server \\
\hline$\|$ & Concatenation operation \\
\hline App_Auth_Ack & Application authentication acknowledgment message \\
\hline$P R_{A P P}$ & Private key of the application server \\
\hline$P U_{A P P}$ & Public key of the application server \\
\hline$a$ and $D P_{a}$ & Device's elliptic curve Diffie-Hellman private and public keys \\
\hline$b$ and $D P_{b}$ & Application server's elliptic curve Diffie-Hellman private and public keys \\
\hline & Elliptic curve Diffie-Hellman base point \\
\hline $\operatorname{pad}_{16}$ & Function adding zero octets to make the length of the data a multiple of 16 \\
\hline hoal [.] & hval refers to hash value and [.] to the index. \\
\hline
\end{tabular}

Its network architecture consists of four entities: device (sensors), gateway, network server and application server. As illustrated in Figure 1, each device is connected to its network server via the corresponding gateway(s) where the device-gateway path is over a single wireless hop and the gateway-network server is interconnected with the non-LoRaWAN network (IP connections). Like the gateway-network server path, the network server communicates with the application servers via a non-LoRaWAN network (IP connections).

\subsection{Standard LoRaWAN Protocol}

In the LoRaWAN network, each device needs to perform a join procedure to enter into the network. The join procedures are classified as Over-The-Air Activation (OTAA) and Activation By Personalization (ABP).

\subsubsection{Over-the-Air Activation and Activation by Personalization}

In the OTAA mode, a device and its network server mutually authenticate each other and exchange the network and application session keys, NwkSKey and AppSKey, through the Join procedure. Among the exchanged session keys, the application key AppSKey is forwarded to the corresponding application server so that the device and the application server securely exchange data. On the other hand, in ABP mode, it is assumed that the two session keys, NwkSKey and AppSKey, are stored on their device with the device address DevAddr. Therefore, each device can immediately start to communicate with its 
application server via the LoRaWAN network while skipping the join procedure. This paper focuses on OTAA mode.

\subsubsection{Join Procedure}

The join procedure is depicted in Figure 2. In order to enter the LoRaWAN network, the device starts the join procedure by sending the Join_Request message. Prior to this, it first randomly generates DevNonce and then computes $M I C_{1}$ with the long-term secret key AppKey. Upon receiving the message, the network server verifies the included $M I C_{1}$. If positive, it gains trust for the device and then randomly generates AppNonce to proceed to the next steps. Afterwards, the network server prepares for the Join_Accept message by computing $M I C_{2}$ and encrypting the message AppNoncei ||$N e t I D\|D e v A d d r|| R F U\| R x$ Delay $\| C F L i s t|| M I C_{2}$ with AppKey. At the same time, it makes the two session keys AppSKey $=E\left(\right.$ AppKey, OX01\|AppNonce $\|$ NetID $\|{\left.\text { DevNonce } \| p a d_{16}\right)}$ and NwkSKey $=E\left(\right.$ AppKey,0X02\|AppNonce $\|$ NetID $\|$ DevNonce $\|$ pad $\left.{ }_{16}\right)$. The network server concludes the join procedure by sending the Join_Accept message to the device and forwarding AppSKey to the application server. On receipt of the message, the device decrypts it and verifies the included $M I C_{2}$. If the verification is successful, the network server is authenticated to the device, which then generates the two session keys, AppSKey and NwkSKey. As a result, the device and the network server mutually authenticate each other while exchanging the two session keys. In addition, AppSKey is shared between the device and the application server.

\section{Secure Channel: - - -}

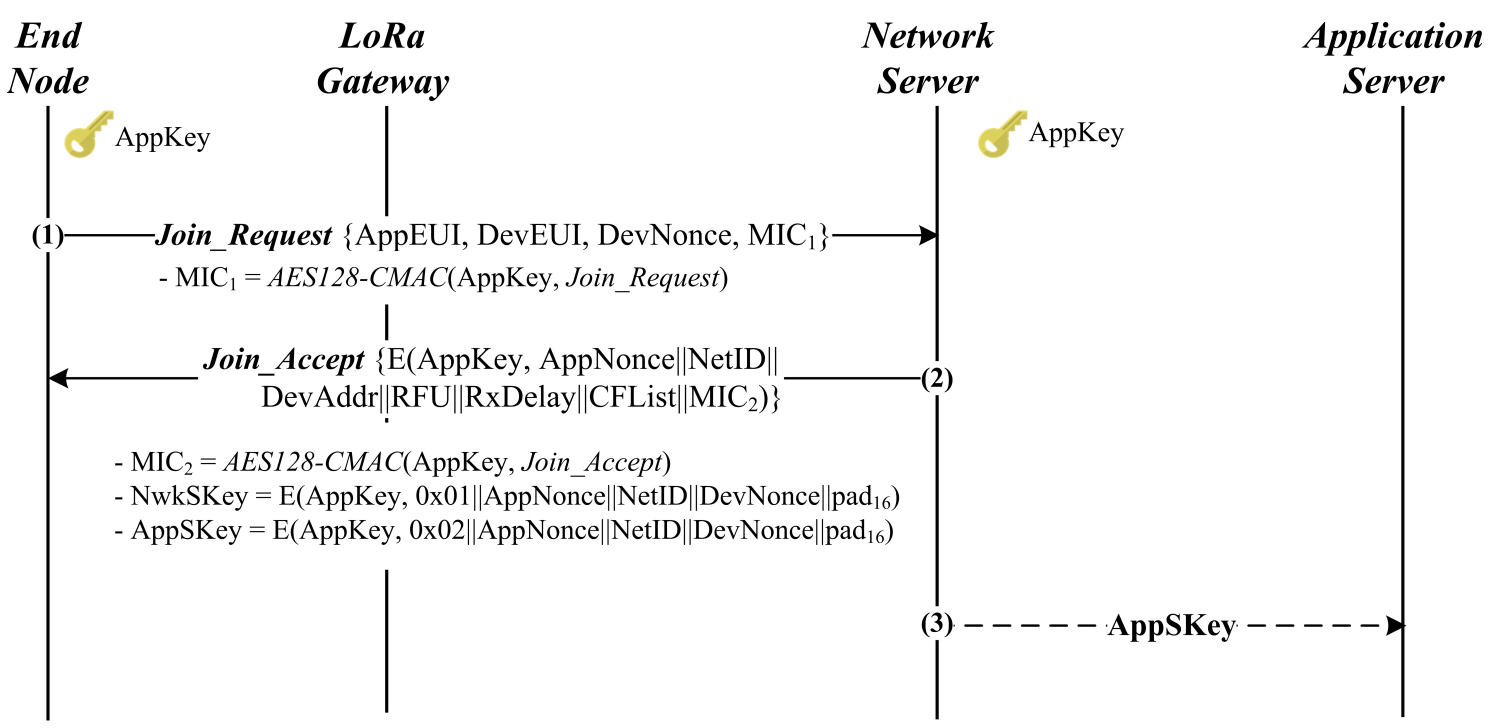

Figure 2. LoRaWAN Join procedure.

\subsection{Problems with the Standard LoRaWAN Protocol}

The standard LoRaWAN protocol faces the following problems irrespective of its preliminary securities:

- There is no prevention against the replay attack in the Join_Request and Join_Accept messages because the device and the network server cannot accept the freshness of AppNonce and DevNonce, respectively.

- The end-to-end security between the device and the application server is broken because AppSKey is known to the network server. 
- The session keys are derived from the long-term secret key AppKey. Therefore, if AppKey is compromised, the past session keys can be recovered while the encrypted traffic can be decrypted, i.e., the perfect forward secrecy does not hold.

\section{Related Work}

As mentioned above, because the network server generates two session keys, network operators are able to decrypt and intercept all the data passing through the network server. Girard [52] pointed out this problem and suggested to deploy the trusted third party to enhance the LoRaWAN network. According to the LoRaWAN specification v1.02 [51], it is clearly defined that compromising the keys of the one device does not impact the other ones' secure communication. However, in ABP mode, the keys are derived from the device address, which leads to a vulnerability with reverse engineering [40]. Further, with the standard protocol, there exists a loophole of the end-to-end security and the vulnerability to the replay attack.

Avoine and Ferreira [53] introduced several attacks that affect the network availability, data integrity and data confidentiality in the earlier versions of LoRaWAN. The authors emphasized the replay or decrypt attack and desynchronization attack. These attacks are discussed by considering an end-device or the network server as the target entity. In the replay attack scenario, the authors discuss two techniques for attack: replay of a Join_Accept message and harvest of Join messages. Similarly, in the desynchronization attack, the target can be an end device or the network server, which is responsible for disconnecting the end-device from the network. By considering these attacks, the authors recommended that AppNonce value should follow freshness, provide the detection mechanism against the replay attacks, verify that the received Join_Accept message corresponds to the sent Join_Request message and also check that session keys are shared or not.

Kim and Song [42] tried to provide end-to-end security between the device and the application server by allowing the two parties to directly negotiate AppSKey without involving the network server. However, it needs to change the standard, which makes its application difficult in the existing LoRaWAN network. Moreover, this approach cannot provide the perfect forward secrecy.

$\mathrm{Na}$ et al. [41] introduced an effective countermeasure against the replay attack in the join procedure. In more detail, this approach uses eXclusive-OR DevNonce with AppKey or the previous session key to make the Join_Request message fresh. Even though this approach effectively addresses the replay attack, it does not support the end-to-end security, nor the perfect forward secrecy.

In Garcia et al. (radius-based) [44], an explicit entity termed as the join server is used, which is responsible for authentication of devices. The join server plays the role of an external Authentication, Authorization, and Accounting (AAA) server. In this approach, the AAA server instead of the network server mutually authenticates the device while exchanging the session keys. With the integration of the LoRaWAN joining procedure with the radius mechanism, the AAA server can make the network server free from the key management overheads. However, even in such a scenario, the network server knows all the keys, thus being able to decrypt all the packets between the device and the application server (i.e., the end-to-end security is broken). Moreover, a long latency is also caused because of the involvement of the AAA server. Further, this scheme does not focus on the replay attack problem in the join request.

In Garcia et al. (diameter-based) [43], the network server serves as the diameter client to communicate with the AAA server. On receiving the join request from the device, the network server counts on the AAA server to authenticate the device and generate the session keys. Similar to the radius-based approach, this approach can make the network server free from the key management overheads. However, because the network server knows all the keys, it can decrypt and understand all the packets between the device and the application server (i.e., the end-to-end security is broken). In addition, a long latency happens without a focus on the replay attack problem in the join request. A state-of-the-art comparison of different LoRaWAN schemes is presented in Table 2. 
Table 2. Comparisons of different LoRaWAN schemes.

\begin{tabular}{lllllll}
\hline & Standard & Na & Kim and & Garcia & Garcia & Proposed \\
LoRaWAN [51] & et al. [41] & Song [42] & et. [43] & et al. [44] & Po-SEO \\
\hline Scheme & Standard & XoR & Dual & Diameter & Radius & DOS \\
Mutual Authentication & YES & YES & YES & YES & YES & YES \\
Secure Key Exchange & YES & YES & YES & YES & YES & YES \\
Perfect Forward Secrecy & NO & NO & NO & NO & NO & YES \\
End-to-End Security & NO & NO & NO & NO & NO & YES \\
Defense against a Replay Attack & NO & YES & NO & NO & NO & YES \\
\hline
\end{tabular}

\section{Enhanced LoRaWAN Security Protocol}

This section presents the enhanced LoRaWAN security protocol, which addresses the standard one's security flaws. It not only suggests the DevNonce generation method to prevent the replay attack, but also enables a device and its application server to achieve the true end-to-end security through the Elliptic Curve Diffie Hellman (ECDH)-based key exchange [54], which is authenticated by the Elliptic Curve Digital Signature Algorithm (ECDSA) [55]. Especially, the presented protocol provides two options, the Default Option (DO) and the Security-Enhanced Option (SEO), to prevent a malicious network server from breaking the end-to-end security between a device and its application server. The first option DO aims to defend against a malicious network server attempting to eavesdrop on the communication between a device and its application server. In the second option SEO, a malicious network server is blocked from manipulating packets between a device and its application server, as well as impersonating these two parties.

The proposed protocol has the following assumptions: (i) AppKey is a long-term secret shared between a device and its network server. (ii) A secure channel is pre-established between the network and application servers. (iii) Every device has and trusts its application server's ECDSA public key $P U_{A P P}$, which is used to verify the application server's digital signature. (iv) In the case of SEO, every device should have its own ECDSA public key pair, and its public key $P U_{D E V}$ should be trusted by the application server. How $P U_{A P P}$ and $P U_{D E V}$ are safely delivered, revoked and updated is beyond this paper's scope. Here, the LoRa gateway is skipped because it has no contribution to security.

\subsection{Default Option}

Figure 3 outlines the first option DO, which is composed of six steps. In the first two steps, the device and the network server authenticate each other while exchanging two session keys. Then, the device and the application server perform mutual authentication, as well as establish the strong session key, $S K$, based on which, both the end-to-end security and the perfect forward secrecy hold.

Details on this option are described as follows.

(1) A device attempts to enter the LoRaWAN network by sending the Join_Request message. To protect this message, the device generates the $i$-th fresh nonce DevNonce ${ }_{i}$ and the message integrity code $M I C_{1}$. Figure 4 shows how the DevNonce $e_{i}$ is computed in the way of making the Join_Request message fresh, which enables this step to prevent replay attacks. In addition, $M I C_{1}$ is obtained by computing AES128 - CMAC(AppKey, Join_Request).

(2)-(3) On receiving the Join_Request message, the network server verifies if the received DevNonce $_{i}$ and $M I C_{1}$ are correct. In the positive case, the device is successfully authenticated to the network server, which then prepares for the network server's $i$-th nonce AppNonce $e_{i}$ by eXclusive-ORing a randomly generated nonce AppNonce with the received DevNonce ${ }_{i}$, generates two session keys AppSKey and NwkSKey and computes $M I C_{2}$. Note that $A p p N o n c e_{i}$ can guarantee the device the Join_Accept message's freshness and relation to the received Join_Request message. Finally, the network server encrypts with AppKey all the message contents including AppNonce ${ }_{i}$, NetID, DevAddr, RFU, RxDelay, CFList and $M I C_{2}$ into the Join_Accept message, which is then sent to the device. At the 
same time, the network server forwards the application server the newly-generated session key AppSKey so that the App_Auth_Req message can be safely exchanged with that key. Upon a receipt of the Join_Accept message, the device decrypts it with AppKey and verifies the correctness of the decrypted AppNonce ${ }_{i}$ and $M I C_{2}$. If the verification is successful, the device can conclude that the message is fresh and the network server is authentic, followed by computing the two session keys, AppSKey and NwkSKey. At this point, it is worth noting that this protocol provides the first two messages' freshness, the mutual authentication between the device and the network server and the session key exchange.

(4) Once having successfully verified the Join_Accept message, the device proceeds with the remaining Steps (4)-(6) by preparing for the App_Auth_Req message. For this message, it first computes $\operatorname{Seq}_{1}=(16$, sha1 $(64, A p p S K e y))$, which is made fresh by being derived from AppSKey. In addition, its ECDH private key $d$ is randomly generated, and the corresponding public key $D P_{d}=d G$ is computed where $G$ is the base point. Finally, the session key AppSKey is applied to compute $M I C_{3}=A E S 128-C M A C\left(A p p S K e y, A p p \_A u t h \_R e q\right)$, which secures both the App_Auth_Req message and the ECDH key exchange.

(5) Upon receiving the App_Auth_Req message, the application server validates $S e q_{1}$ and $M I C_{3}$. If valid, it randomly creates its own ECDH private key $a$ and calculates the corresponding public key $D P_{a}=a G$, followed by obtaining $S K=\operatorname{sha} 1\left(a D P_{d} \| S e q_{2}\right)$. At this point, the application server can defend against resource exhaustion attacks by flooding the App_Auth_Req messages because it first checks $M I C_{3}$ prior to the expensive ECDH operations. Afterwards, it makes $M I C_{4}=E\left(P R_{A p p}, \operatorname{sha1}\left(S_{e q}\|A p p E U I\| D e v E U I\left\|D P_{d}\right\| D P_{a} \| S K\right)\right.$ and $M I C_{5}=A E S 128-C M A C\left(A p p S K e y, A p p \_A u t h \_R e s\right)$, which, together with other values, constitute the $A p p \_A u t h \_$Res message. Note that $M I C_{4}$, which is a digital signature generated with the application server's private key $P R_{A p p}$, plays a role in defending against the man-in-the-middle attacks launched by a malicious network server. The application server finishes Step (5) by responding to the device with the App_Auth_Res message.

(6) After receiving the App_Auth_Res message, the device first verifies the received $S e q_{2}$ and $M I C_{5}$. If they are valid, the device trusts that the received message is fresh and protected by AppSKey. Such a trust allows the device to establish the session key $S K$ without being vulnerable to the replay and resource exhaustion attacks. Then, the device validates $M I C_{4}$ with the application's public key $\mathrm{PU}_{A P P}$. If $M I C_{4}$ is correct, the application server is authenticated to the device, which then concludes the proposed protocol by sending the App_Auth_Ack message. At this point, the device can prevent the man-in-the-middle attacks by a malicious network server because $M I C_{4}$ can only be generated by the application server. Once the message arrives, the application server attempts to verify if it is fresh and valid with the received $\mathrm{Seq}_{3}$ and $M I C_{6}$. If this verification is successful, the device is authenticated to the application server, as well as shown to have $S K$.

It is worth noting that the first option DO counts on the digital signature $M I C_{4}$ to block a malicious network server from carrying out the man-in-the-middle attack on the ECDH-based key exchange. That makes it impossible for the malicious server to interpret and manipulate the packets transmitted between the device and the application server. However, this option is still vulnerable to the impersonation attack that the malicious network server can launch by forging the App_Auth_Req message with AppSKey. 
Secure Channel: - - - -

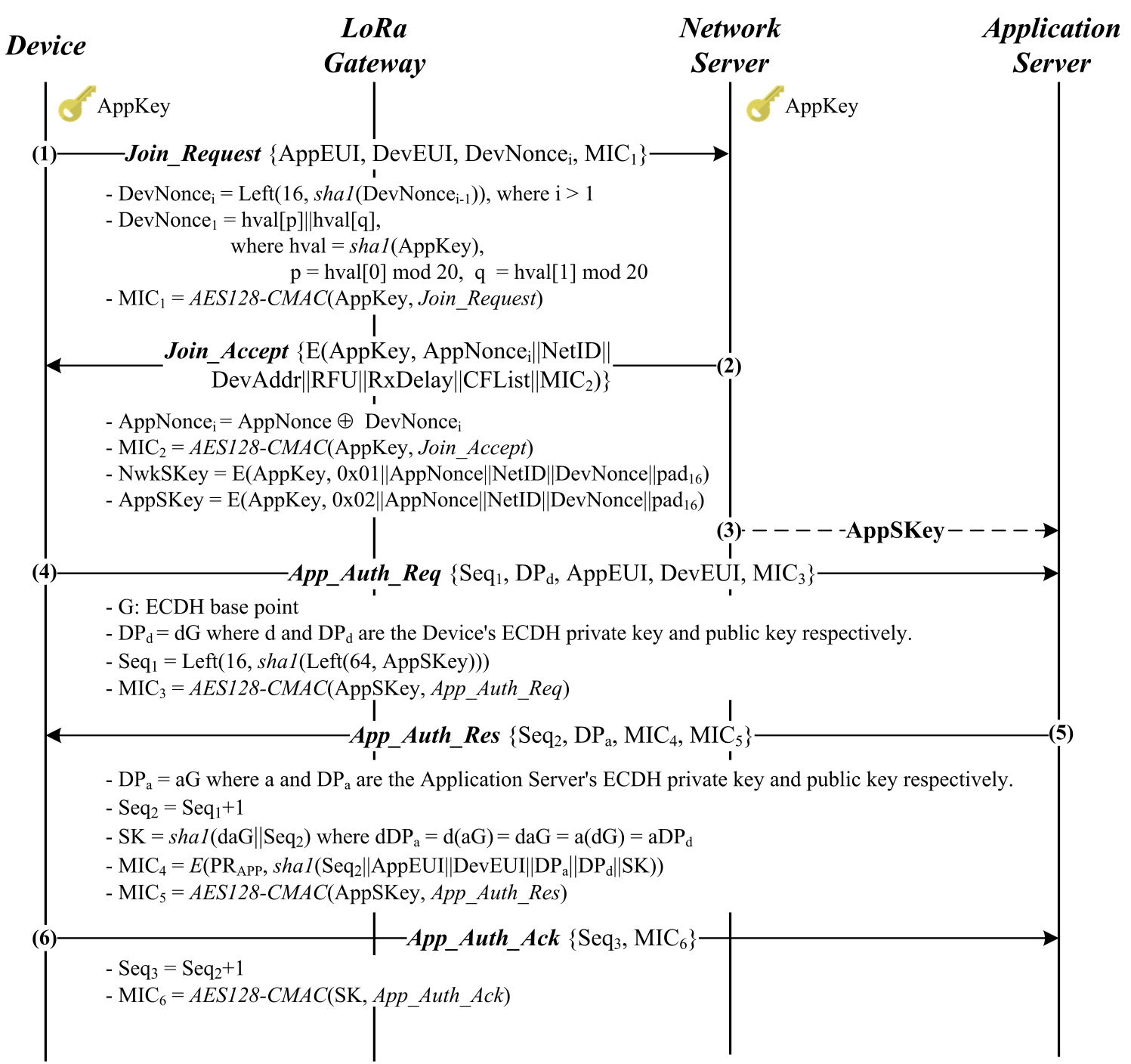

Figure 3. Proposed protocol's default option.

$$
\begin{aligned}
& \text { DevNonce }_{\mathrm{i}}=\operatorname{Left}\left(16, \text { shal }_{\left.\left(\text {DevNonce }_{\mathrm{i}-1}\right)\right)}\right) \text {, where } \mathrm{i}>1 \\
& \text { DevNonce }_{1}=\text { hval[p] } \mid \text { hval[q], } \\
& \text { where hval }=\operatorname{shal}(\text { AppKey }), / / \text { hval }=\text { hval[0]...hval[19] } \\
& \mathrm{p}=\mathrm{hval}[0] \bmod 20, \mathrm{q}=\mathrm{hval}[1] \bmod 20
\end{aligned}
$$

Figure 4. DevNonce generation.

\subsection{Security-Enhanced Option}

The second option SEO aims at defeating the impersonation attack mentioned above while keeping the DO's security properties. Therefore, as shown in Figure 5, this option's messages are exactly the same as those of DO except for the $A p p \_A u t h \_$Req message. In order to prevent the impersonation attack by the malicious network server, the App_Auth_Req message includes the digital signature $M I C_{3 a}$ generated with the device's ECDSA private key, $P R_{D E V}$. This signature disables the network server to masquerade as the device by using AppSKey. Here, we assume that with the given 
DevEUI, the application server can securely retrieve the device's ECDSA public key, $P U_{D E V}$, from its centralized repository rather than directly from the device.

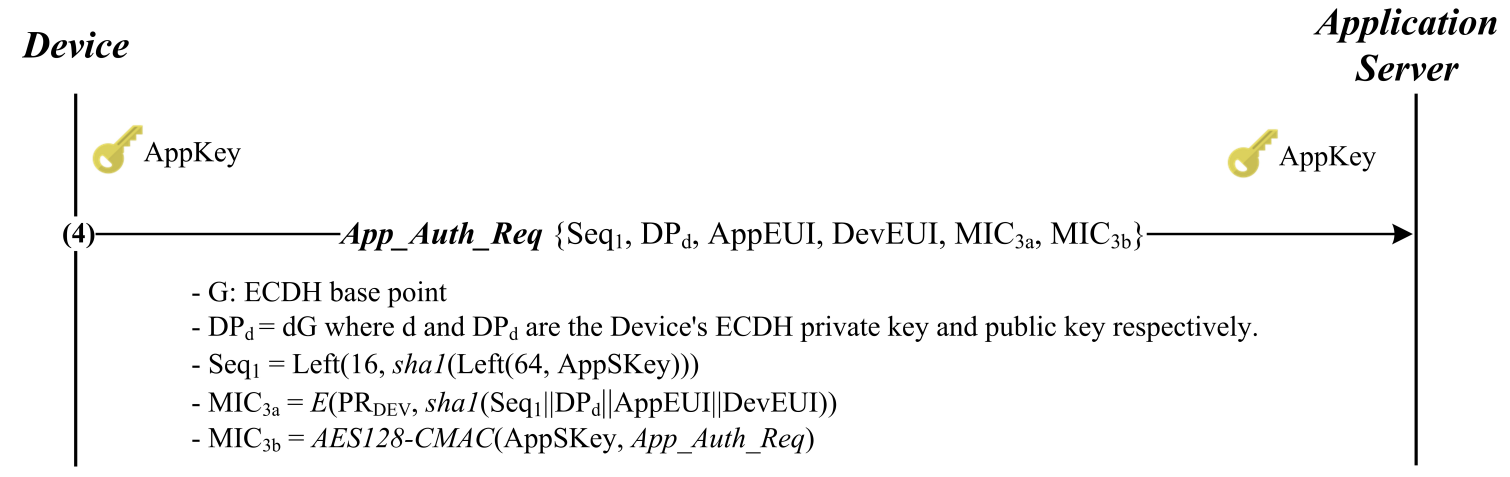

Figure 5. Proposed protocol's security-enhanced option.

\section{Security Analysis}

In this section, the proposed protocol is formally analyzed through BAN logic [10,46,56,57], and then, the possibility of attack is thoroughly examined by the AVISPA tool.

\subsection{BAN Analysis}

According to the typical BAN logic analysis, the proposed protocol is first idealized; its assumptions and goals are defined, and then, the belief derivation is repeatedly conducted until obtaining the intended results. The BAN logic's notations and rules are shown in Table 3 and Figure 6.

Table 3. Burrows-Abadi-Needham (BAN)-logic notations.

\begin{tabular}{ll}
\hline Statement & Meaning \\
\hline$P$ believes $X$ & $P$ believes $X$ and acts as if $X$ were true. \\
$P$ sees $X$ & $P$ receives $X$ at present or in the past time. \\
$P$ said $X$ & $P$ once said $X$, which means that $X$ was sent to $P$ at some point. \\
$P$ controls $X$ & $P$ has jurisdiction over $X$. \\
$\#(X)$ & $X$ is fresh. \\
$\{X\}_{K}$ & $X$ is encrypted with a secret $K$. \\
$\langle X\rangle_{K}$ & It means that $X$ is combined with secret $K$. MIC can be expressed by this notation. \\
$P \stackrel{K}{\leftrightarrow} Q$ & $K$ is a secret key only known to $P$ and $Q$. \\
$\stackrel{K}{\leftrightarrow} P$ & $K$ is $P^{\prime}$ s public key. \\
$P \stackrel{K}{\Leftrightarrow} Q$ & $K$ is a secret only known to $P$ and $Q$. \\
\hline
\end{tabular}

\subsubsection{Default Option}

At first, the default option DO is translated into an idealization as follows (where $D, N S$ and APP denote device, network server and application server, respectively):

(1) $\left.\langle\text { AppEUI,DevEUI,DevNonce }\rangle_{i}\right\rangle_{\text {AppKey }}$

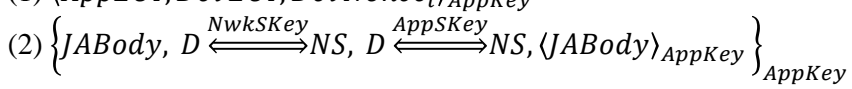

where JABody $=\left\{\right.$ AppNonce $_{i}$, NetID, DevAddr, RFU, RxDelay, CFList $\}$

(3) $\left\langle\text { Seq }_{1}, D P_{d}, A p p E U I, D e v E R I\right\rangle_{A p p S K e y}$

(4) $\left\langle\operatorname{Seq}_{2}, D P_{a},\left\{\stackrel{S K}{\Leftrightarrow} A P P, D \stackrel{S K}{\leftrightarrow} A P P, S e q_{2}, A p p E U I, D e v E U I, D P_{d}, D P_{a}\right\}_{P U_{A P P}^{-1}}\right\rangle_{A p p S K e y}$

(5) $\left\langle\text { Seq }_{3}, D \stackrel{S K}{\Leftrightarrow} A P P, D \stackrel{S K}{\leftrightarrow} A P P\right\rangle_{S K}$ 


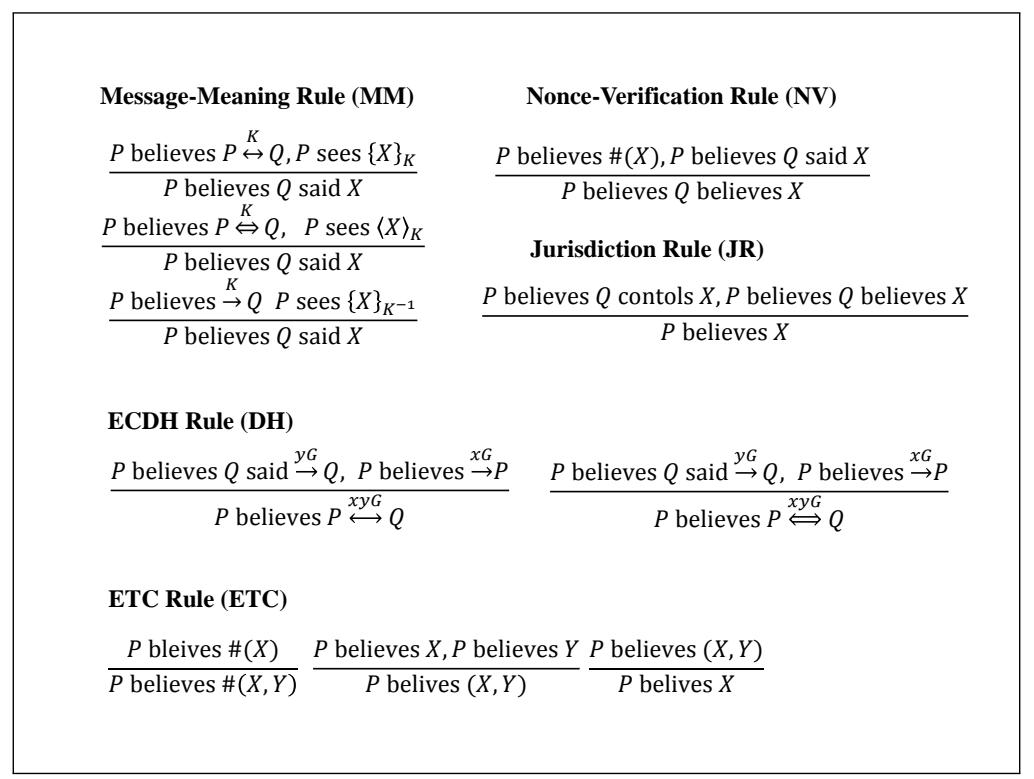

Figure 6. BAN logic rules.

As the next step, it is necessary to make the DO's assumptions and goals. The assumptions are as follows:
(A1) NS believes D $\stackrel{\text { AppKey }}{\Longleftrightarrow} \mathrm{NS}$
(A2) NS believes \# (DevNonce $\left.{ }_{i}\right)$
(A3) NS believes $\mathrm{D} \stackrel{\text { NwkSKey }}{\Longleftrightarrow}$ NS
(A4) NS believes D $\stackrel{\text { AppSKey }}{\rightleftarrows}$ NS
(A5a) D believes D $\stackrel{\text { AppKey }}{\longleftrightarrow}$ NS
(A5b) D believes $\mathrm{D} \stackrel{\text { AppKey }}{\Longleftrightarrow} \mathrm{NS}$
(A6) D believes \# (AppNonce ${ }_{i}$ )
(A7) D believes NS controls $\mathrm{D} \stackrel{K}{\Leftrightarrow} \mathrm{NS}$

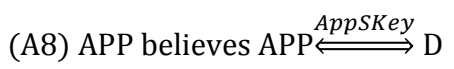
(A9) APP believes \#(Seq $)$
(A10) APP believes $\stackrel{D P_{a}}{\longrightarrow}$ APP
(A11) D believes \# $\left(\mathrm{Seq}_{2}\right)$
(A12) D believes $\stackrel{D P_{d}}{\longrightarrow} \mathrm{D}$
(A13) D believes $\stackrel{P U_{A P P}}{\longrightarrow}$ APP
(A14) APP believes \# $\left(\mathrm{Seq}_{3}\right)$

Strictly speaking, (A8) is not reasonable because AppSKey is known to the network server in addition to the device and the application server. However, DO does not consider the malicious network server trying to impersonate the device by forging the App_Auth_Req message. Therefore, (A8) is maintained to reason about DO under such an attacker model.

The goals are as follows:
(G1) NS believes D believes (AppEUI, DevEUI)
(G2) D believes NS believes JABody
(G9) D believes D $\stackrel{S K}{\Leftrightarrow} \mathrm{APP}$
(G3) D believes D $\stackrel{\text { NwkSKey }}{\Longleftrightarrow} \mathrm{NS}$
(G4) D believes D $\stackrel{\text { AppSKey }}{\Longleftrightarrow}$ NS
(G5) D believes NS believes $\mathrm{D} \stackrel{\text { NwkSKey }}{\Longleftrightarrow} \mathrm{NS}$
(G10) D believes D $\stackrel{S K}{\leftrightarrow} \mathrm{APP}$
(G11) D believes APP believes D $\stackrel{S K}{\Leftrightarrow}$ APP
(G12) D believes APP believes D $\stackrel{S K}{\leftrightarrow}$ App
(G6) D believes NS believes D $\stackrel{\text { AppSKey }}{\Longleftrightarrow \mathrm{NS}}$
(G13) APP believes APP $\stackrel{S K}{\Leftrightarrow} \mathrm{D}$
(G14) APP believes APP $\stackrel{S K}{\leftrightarrow} \mathrm{D}$
(G7) D believes APP believes (AppEUI, DevEUI)
(G8) APP believes D believes $\mathrm{Seq}_{3}$
(G15) APP believes D believes D $\stackrel{S K}{\Leftrightarrow}$ APP
(G16) APP believes D believes D $\stackrel{S K}{\leftrightarrow}$ APP

In the above goals, (G1) and (G2) indicate the mutual authentication between the device and the network server, while (G7) and (G8) indicate the mutual authentication between the device and the 
application server. On the other hand, (G3)-(G6) mean that the device obtains the belief that it shares the two session keys, NwkSKey and AppSKey, with the network server. In addition, the remaining goals express that the session key $S K$ is securely exchanged between the device and the application server. With the above-idealized version, assumptions and goals, the formal analysis proceeds.

From (1), we derive:

(D1) NS sees $\left\langle A p p E U I, D e v E U I, \text { DevNonce }_{i}\right\rangle_{\text {AppKey }}$

(D2) NS believes D said (AppEUI,DevEUI, DevNonce ${ }_{i}$ ) by (D1), (A1), MM

(D3) NS believes D believes (AppEUI, DevEUI, DevNonce $e_{i}$ ) by (D2), (A2), ETC, NV

(D4) NS believes D believes (AppEUI, DevEUI) by (D3), ETC

From (2), we derive:

(D5) D sees $\left\{\text { JABody, D } \stackrel{\text { NwkSKey }}{\Longleftarrow} \mathrm{NS}, \mathrm{D} \stackrel{\text { AppsKey }}{\Longleftrightarrow} \mathrm{NS},\langle\mathrm{JABody}\rangle_{\text {AppKey }}\right\}_{\text {AppKey }}$

(D6) D believes NS said (JABody, D $\stackrel{\text { NwkSKey }}{\Longleftrightarrow} \mathrm{NS}, \mathrm{D} \stackrel{\text { AppSKey }}{\Longleftrightarrow} \mathrm{NS},\langle\mathrm{JABody}\rangle_{\text {AppKey }}$ ) by (D5), (A5a), MM

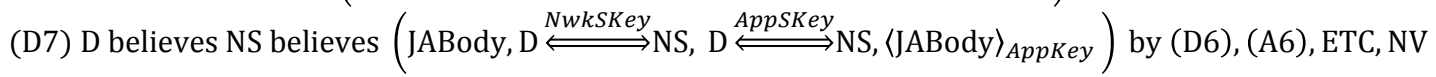

(D8) D believes NS believes JABody by (D7), ETC

(D9) D believes NS believes D $\underset{\text { AppSKey }}{\stackrel{\text { NwkSKey }}{\rightleftarrows}} \mathrm{NS}$ by (D7), ETC

(D10) D believes NS believes D $\stackrel{\text { AppsKey }}{\Longleftrightarrow}$ NS by (D7), ETC

(D11) D believes NS believes JABody by (D7), ETC, (A5b), MM, (A6), ETC, NV

(D12) D believes D $\stackrel{\text { NwkSKey }}{\Longleftarrow} \mathrm{NS}$ by (D9), (A7), JR

(D13) D believes D $\stackrel{\text { AppSKey }}{\Longleftrightarrow} \mathrm{NS}$ by (D10), (A7), JR

From (3), we derive:

(D14) APP sees $\left\langle S e q_{1}, D P_{d}, A p p E U I, D e v E R I\right\rangle_{\mathrm{AppSKey}}$

(D15) APP believes D believes ( $\left.S e q_{1}, D P_{d}, A p p E U I, D e v E R I\right)$ by (D14), (A8), MM, (A9), ETC, NV

(D16) APP believes D believes (AppEUI,DevERI) by (D15), ETC

(D17) APP believes D believes $D P_{d}$ by (D15), ETC

(D18) APP believes APP $\stackrel{S K}{\leftrightarrow} \mathrm{D}$ by (D17), (A10), DH where $S K=a D P_{d}=a d G$

(D19) APP believes APP $\stackrel{S K}{\Leftrightarrow}$ D by (D17), (A10), DH

From (4), we derive:

(D20) D sees $\left\langle S e q_{2}, D P_{a},\left\{\mathrm{D} \stackrel{S K}{\Leftrightarrow} \mathrm{APP}, \mathrm{D} \stackrel{S K}{\leftrightarrow} \mathrm{APP}, S e q_{2}, A p p E U I, D e v E U I, D P_{d}, D P_{a}\right\}_{P U_{A P P}^{-1}}\right\rangle_{\mathrm{AppSKey}}$

(D21) D believes APP believes $\left(S e q_{2}, D P_{a},\left\{\mathrm{D} \stackrel{S K}{\Leftrightarrow} \mathrm{APP}, \mathrm{D} \stackrel{S K}{\leftrightarrow} \mathrm{APP}, S e q_{2}, A p p E U I, \operatorname{DevEUI}, D P_{d}, D P_{a}\right\}_{P U_{A P P}^{-1}}\right)$ by (D20), (D13), MM, (A11), ETC, NV

(D22) D believes APP believes $D P_{a}$ by (D21), ETC

(D23) D believes D $\stackrel{S K}{\Leftrightarrow} \mathrm{APP}$ by (D22), (A12), DH where $S K=d D P_{a}=a d G$

(D24) D believes D $\stackrel{S K}{\leftrightarrow}$ APP by (D22), (A12), DH

(D25) D sees $\left\{\mathrm{D} \stackrel{S K}{\Leftrightarrow} \mathrm{APP}, \mathrm{D} \leftrightarrow \mathrm{APP}, S e q_{2}, A p p E U I, D e v E U I, D P_{d}, D P_{a}\right\}_{P U_{A P P}^{-1}}$ by (D21), ETC

(D26) D believes APP believes ( $\left.\mathrm{D} \stackrel{S K}{\Leftrightarrow} \mathrm{APP}, \mathrm{D} \stackrel{S K}{\leftrightarrow} \mathrm{App}, S e q_{2}, A p p E U I, D e v E U I, D P_{d}, D P_{a}\right)$

(D27) D believes APP believes D $\stackrel{S K}{\Leftrightarrow}$ APP by (D26), ETC by (A13), (D25), MM, (A11), ETC, NV

(D28) D believes APP believes D $\stackrel{S K}{\leftrightarrow}$ App by (D26), ETC

(D29) D believes APP believes (AppEUI,DevEUI) by (D26), ETC 
From (5), we derive:

(D30) APP sees $\left\langle\mathrm{Seq}_{3}, \mathrm{D} \stackrel{S K}{\Leftrightarrow} \mathrm{APP}, \mathrm{D} \stackrel{S K}{\leftrightarrow} \mathrm{APP}\right\rangle_{\mathrm{SK}}$

(D31) APP believes D believes ( $\left.S e q_{3}, \mathrm{D} \stackrel{S K}{\Leftrightarrow} \mathrm{APP}, \stackrel{S K}{\leftrightarrow} \mathrm{APP}\right)$ by (D30), (D19), MM, (A14), ETC, NV

(D32) APP believes D believes D $\stackrel{S K}{\Leftrightarrow}$ APP by (D31), ETC

(D33) APP believes D believes D $\stackrel{S K}{\leftrightarrow}$ APP by (D31), ETC

(D34) APP believes D believes $\mathrm{Seq}_{3}$ by (D31), ETC

Based on the above derivations, we can show that the goals are satisfied as follows, while thus concluding that DO is correct.

- (G1) and (G2) are satisfied by (D4) and (D8), respectively.

- (G3)-(G6) are satisfied by (D12), (D13), (D9) and (D10), respectively.

- (G7) and (G8) are satisfied by (D29) and (D34), respectively.

- (G9)-(G16) are satisfied by (D23), (D24), (D27), (D28), (D18), (D19), (D32) and (D33), respectively.

\subsubsection{Security-Enhanced Option}

In order to analyze SEO, we focus on only the App_Auth_Req message because DO and SEO are the same except for it. Therefore, the $A p p \_A u t h \_$Req message is idealized as follows:

(3) $\left\langle\operatorname{Seq}_{1}, D P_{d}, A p p E U I, D e v E R I,\left\{S e q_{1}, D P_{d}, A p p E U I, D e v E R I\right\}_{P U_{D E V}^{-1}}\right\rangle_{A p p S K e y}$

The assumption (A15) and the goal (G17) are added as follows:

$$
\text { (A15) APP believes } \stackrel{P U_{D E V}}{\longrightarrow} \mathrm{D} \quad \text { (G17) APP believes D believes } S e q_{1}
$$

Here, like (G8), (G17) indicates that the device is authenticated to the application server. In other words, (G8) is complemented by (G17), which is obtained through ECDSA.

From (3), we derive:

(D35) APP sees $\left\langle S e q_{1}, D P_{d}, A p p E U I, D e v E R I,\left\{S e q_{1}, D P_{d}, A p p E U I, D e v E R I\right\}_{P U_{D E V}^{-1}}\right\rangle_{\mathrm{AppSKey}}$

(D36) APP believes D believes (Seq $\left., D P_{d}, A p p E U I, D e v E R I,\left\{S e q_{1}, D P_{d}, A p p E U I, D e v E R I\right\}_{P U_{D E V}^{-1}}\right)$ by (D35), (A8), MM, (A9), ETC, NV

(D37) APP believes D believes (AppEUI, DevERI) by (D36), ETC

(D38) APP believes D believes $D P_{d}$ by (D36), ETC

(D39) APP believes App $\underset{S K}{\leftrightarrow} \mathrm{D}$ by (D38), (A10), DH where $S K=a D P_{d}=a d G$

(D40) APP believes App $\stackrel{S K}{\Leftrightarrow} \mathrm{D}$ by (D38), (A10), DH

(D41) APP sees $\left\{\mathrm{Seq}_{1}, D P_{d}, A p p E U I, D e v E R I\right\}_{P U_{D E V}^{-1}}$ by (D35), ETC

(D42) APP believes D believes $\left(S e q_{1}, D P_{d}, A p p E U I, D e v E R I\right)$ by (D41), (A15), MM, (A9), ETC, NV

(D43) APP believes D believes $S e q_{1}$ by (D42), ETC

Obviously, the goal (G17) is achieved by obtaining (D43), while (G13) and (G14) are satisfied through $\mathrm{D}(39)$ and (D40). Unlike DO, SEO aims to defeat the malicious network server trying to masquerade as the device with AppSKey. For this goal, the device is authenticated to the application server by using the digital signature $M I C_{3 a}$ computed with the ECDSA private key $P R_{D E V}$. Moreover, AppSKey plays a role in preventing the resource exhaustion attack by allowing the application server to check $M I C_{3 b}$ prior to the expensive operations, i.e., ECDSA digital signature verification and ECDH key exchange. Thus, it is still reasonable to maintain (A8). As a result, we can conclude that SEO is correct. 


\subsubsection{Security Properties}

Mutual authentication: The proposed protocol provides the two mutual authentications, i.e., the one between the device and the network server and the other between the device and the application server. Here, by presenting the two lemmas, Lemma 1 and Lemma 2, we prove that the two mutual authentications hold in the proposed protocol.

Lemma 1. The device and network server mutually authenticate each other.

Proof. It is demonstrated from (D4) and (D11) that the device and the application authenticate each other. Therefore, the proposed protocol satisfies the mutual authentication between the device and the application server.

Lemma 2. The device and application server mutually authenticate each other.

Proof. This mutual authentication needs to be proved considering the two options DO and SEO. In the DO case, the proof relies on (D29) and (D34) to show that the device and the application authenticate each other. Note that DO allows (A8) and (D17) because of not taking into consideration the malicious network server impersonating a device. Consequently, the mutual authentication is achieved in DO. On the other hand, in the SEO case, it can be shown from (D29) and (D43) that the mutual authentication between the two parties is satisfied. Clearly, these two obtained beliefs indicate that it is impossible for the malicious network server to launch the man-in-the-middle and impersonate attacks and break the end-to-end security. As a result, it is concluded that the proposed protocol achieves the mutual authentication between the device and the application server.

Secure key exchange: In the proposed protocol, three session keys including NwkSKey, AppSKey and $S K$ are exchanged where NwkSKey is shared between the device and the network server, as well as both AppSKey and SK are shared between the device and the application server. Here, we provide Lemma 3 and Lemma 4 to prove that these keys are securely exchanged.

Lemma 3. NwkSKey and AppSKey are securely exchanged between the device and network server.

Proof. Based on (D9), (D10), (D12) and (D13), we can verify that the session key exchange is authenticated to the device. On the other hand, it can be shown from (D4), (A3) and (A4) that the network server validates the session key exchange. As a result, we can reason that NwkSKey and AppSKey are securely exchanged between the two parties. Note that once the session key exchange is successfully performed, AppSKey is forwarded to the application server. Thus, AppSKey is finally shared between the device and the application server. This session key is properly used according to the selected option, i.e., DO or SEO, so that the security threats caused by the malicious network server can be avoided.

Lemma 4. SK is securely exchanged between the device and application server.

Proof. In the DO case, it can be seen from (D18), (D19), (D23), (D24), (D27), (D28), (D32) and (D33) that $S K$ is securely exchanged between the device and the application server. In the SEO case, the secure key exchange between the two parties can be verified based on (D23), (D24), (D27), (D28), (D32), (D33), (D39) and (D40). Consequently, we can conclude that SK is securely established between the device and the application server.

End-to-end security: In the proposed protocol, it is very important to provide the end-to-end security between the device and the application server. AppSKey, which aims to protect the application traffic, cannot keep this security property because it is known to the network server in addition to the two involved parties. To solve this problem, the proposed protocol allows the device and the application 
server to securely share the session key SK by relying on the ECDH-based key exchange and the ECDSA digital signature. Lemma 5 is proposed to prove that the proposed protocol achieves the end-to-end security between the device and the application server.

Lemma 5. The end-to-end security is provided between the device and application server.

Proof. In the DO case, it is shown from (D18), (D19), (D23) and (D24) that SK is established between the device and the application server via the ECDH-based key exchange. Especially, (D17) and (D29) validate that this key exchange can be trusted. Similarly, in the SEO case, (D23), (D24), (D39) and (D40) verify that the device and the application share $S K$ by the ECDH-based key exchange. Such a key exchange is secured according to (D29) and (D43). As mentioned above, SK is exchanged in a way that it is only known by the two parties involved through the ECDH-based key exchange. In other words, the network server cannot decrypt or modify the application traffic once it is encrypted or protected with the session keys derived from SK. Therefore, it can be concluded that the end-to-end security is offered between the device and the application server.

Perfect forward secrecy: Perfect forward secrecy is a security property where the compromise of long-term keys does not cause the past session keys to be exposed [58]. For this property, we take into consideration SK because it is used to encrypt the application traffic. Below, we provide Lemma 6 to testify that the proposed protocol achieves perfect forward secrecy on the application traffic.

Lemma 6. Perfect forward secrecy is provided for SK.

Proof. According to the obtained beliefs (D18), (D19), (D23), (D24), (D39) and (D40), it is verified that $S K$ is securely exchanged between the device and the application server via the ECDH-based key exchange. In addition, we can see from (D17), (D29) and (D43) that the SK exchange is protected through the ECDSA digital signature, thus defending against the man-in-the-middle attack. It is worth noting that the generated ECDH private keys are forgotten after their session. That makes it impossible for the past session keys to be recovered even when long-term keys are compromised. Therefore, it is proven that perfect forward secrecy is provided for $S K$.

Defense against resource exhaustion attack: Here, we provide Lemma 7 to show that the proposed protocol prevents the resource exhaustion attack, which sends many messages requesting the expensive public key operations to cause the application server and the device to uselessly waste their resources.

Lemma 7. The proposed protocol defends against resource exhaustion attack.

Proof. In the proposed protocol, the application server checks $M I C_{3}$ or $M I C_{3} b$ prior to the expensive public key operations. Similarly, the device verifies the digital signature after validating $M I C_{5}$. Therefore, from the derived beliefs (D17), (D22) and (D38), it is demonstrated that the proposed protocol defends against the resource exhaustion attack.

Defense against replay attack: In the proposed protocol, DevNonce ${ }_{i}$ is made fresh according to the DevNonce generation method, while AppNonce $i_{i}$ is made fresh by eXclusive-ORing the randomly-generated nonce AppNonce with DevNonce ${ }_{i}$. Thus, by including these fresh values, the Join_Request and Join_Accept messages are not vulnerable to the replay attack. On the other hand, the $A p p \_A u t h \_R e q, A p p \_A u t h \_R e s$ and $A p p \_A u t h \_A c k$ messages depend on the sequence numbers $S e p_{1}, S_{e} p_{2}$ and $S e p_{3}$ to prevent the replay attack. Especially, Sep $p_{1}$ is guaranteed to be fresh because it is derived from the new session key AppSKey, and the subsequent numbers are generated by increasing their previous number by one. As a result, the proposed protocol is not vulnerable to the replay attack. 


\subsection{AVISPA Analysis}

AVISPA [47] is an automatic tool for modeling and analyzing security protocols. In AVISPA, a security protocol is modeled based on the High-Level Protocol Specification Language (HLPSL) [59] and converted via HLPSL2IF to Intermediate Format (IF). The converted IF version is then formally analyzed through four sub-modules as shown in Figure 7, and the result is derived.

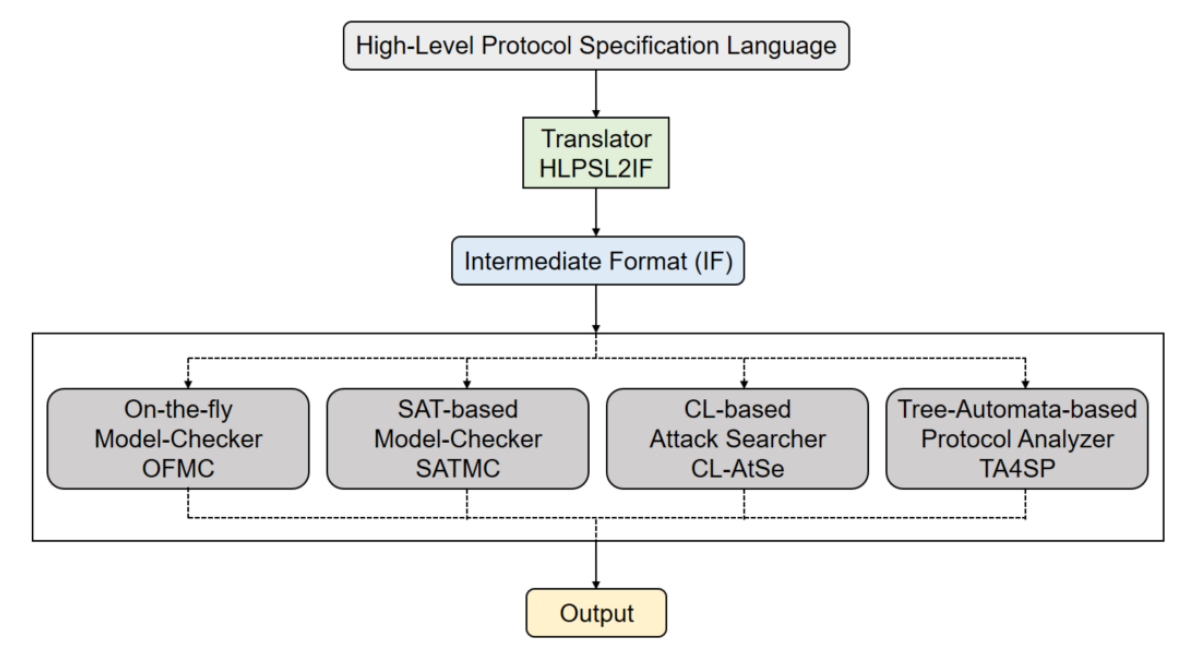

Figure 7. Architecture of Automated Validation of Internet Security Protocols and Applications (AVISPA).

The four sub-modules are as follows:

- OFMC: On-the-Fly Model-Checker [60]

- SATMC: SAT-based Model-Checker [61]

- CL-AtSe: CL-based Attack Searcher [62]

- TA4SP: Tree-Automata-based Protocol Analyzer [63]

\subsubsection{Default Option}

The proposed protocol's DO is modeled in the HLPSL version, which is composed of three roles: the basic, composed and environment roles.

Basic role: The DO's HLPSL version includes the three basic roles, r_Device, r_Network_Server and r_Application_Server, which correspond to a device, network server and application server, respectively. Figure 8 shows the source code of the device's basic role r_Device.

This role possesses the shared key App_Key and its application server's public key PUapp. Furthermore, it communicates with r_Network_Server using the SND_DN and RCV_ND channels while communicating with $\mathrm{r}$ _Application_Server using the SND_DA and RCV_AD channels. Note that all the basic roles including r_Device apply the Dolev-Yao (dy) model, one of the attacker models, to the channels. The basic operations of $r_{-}$Device are defined in the transition section.

In Figure 9, r_Network_Server is defined as a basic role to model the network server. This role shares App_Key and Kna with r_Device and r_Application_Server, respectively. Especially, Kna is used to protect the channel SND_NA between r_Network_Server and r_Application_Server. Moreover, the SND_ND and RCV_DN channels are defined for communication between r_Device and r_Network_Sever. How r_Network_Server works is described in detail in the transition section. The last basic role r_Application_Sever is expressed for the application server as shown in Figure 9. With the symmetric key Kna, this role is able to securely communicate with r_Network_Server over the channel RCV_NA. In addition, the private key corresponding to PUapp, which is expressed as 
auth 3 and auth4, are defined to check if the proposed protocol's authentication holds, followed by the two goals sec1 and sec2, defined for confirming the proposed protocol's secrecy.

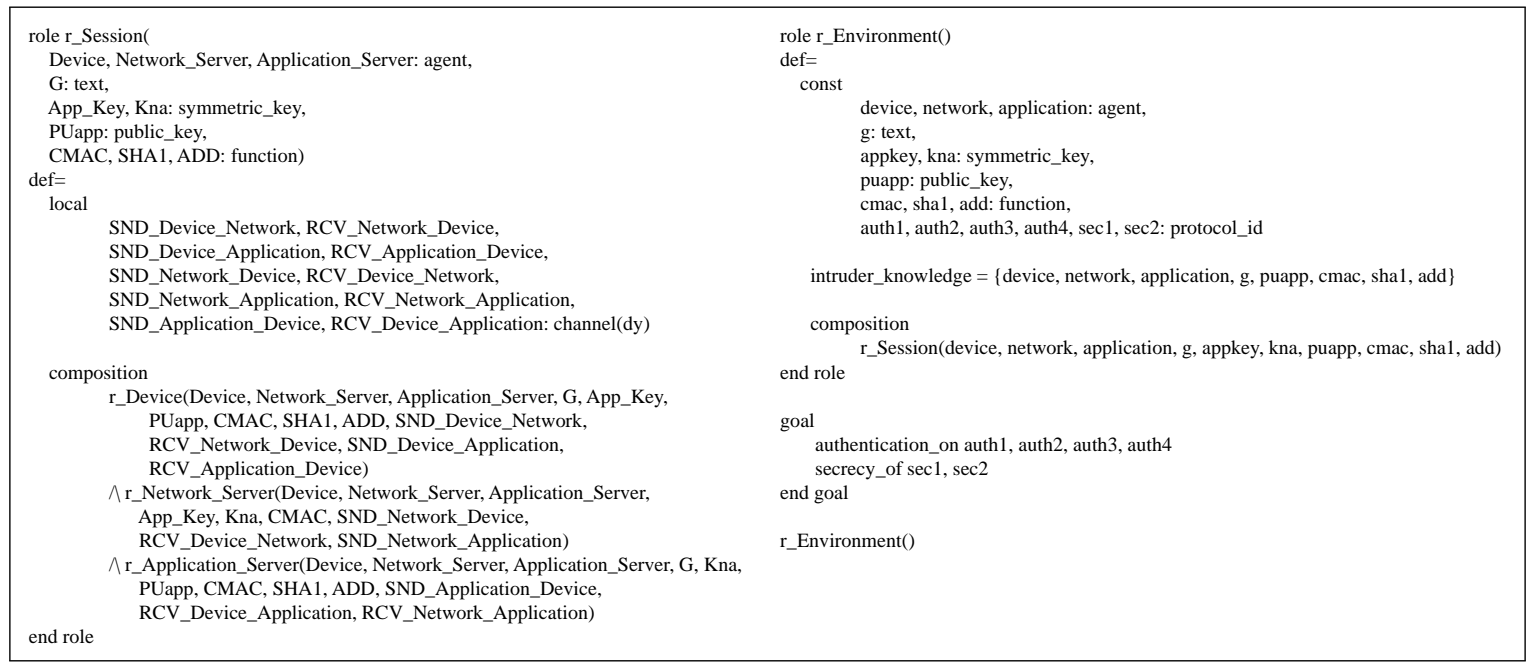

Figure 10. $r \_S e s s i o n$ role and $r \_$Environment role.

Result: Figure 11 shows how the proposed protocol runs in the AVISPA environments. Furthermore, in this figure, we can see the formal verification results on the proposed protocol obtained through the sub-modules of OFCM and CL-AtSe, respectively. According to the results, we can confirm that there is no attack.

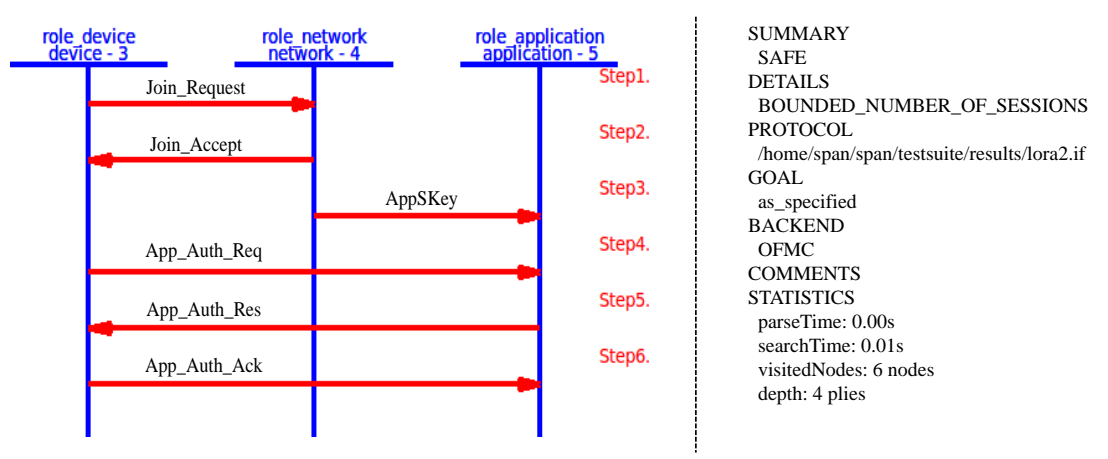

SUMMARY
SAFE
DETAILS
BOUNDED_NUMBER_OF_SESSIONS
TYPED_MODEL
PROTOCOL
/home/span/span/testsuite/results/lora2.if
GOAL
As Specified
BACKEND
CL-AtSe
STATISTICS
Analysed $: 9$ states
Reachable $: 3$ states
Translation $: 0.01$ seconds
Compuatation $: 0.00$ seconds

Figure 11. DO simulation and analysis result.

\subsubsection{Security Enhanced Option}

In SEO, the DO's roles are modified to model the device's digital signature $M I C_{3 a}$ and its related operations. As depicted in Figure 12, the major changes are made in the transitions of r_Device and r_Application. The SEO's simulation and analysis results are exactly the same as those of DO, indicating that no attack is found.

\section{Performance Evaluation}

Initial evaluations are conducted to calculate the message size and the overheads involved in the proposed protocol during communication between the end-to-end devices through coding over real hardware. Next, the two options of the proposed protocol, DO and SEO, are analyzed for their performance by using numerical simulations in comparison with the DTLS-PSK and DTLS-ECC protocols through a case study on a smart factory-enabled parking system. 


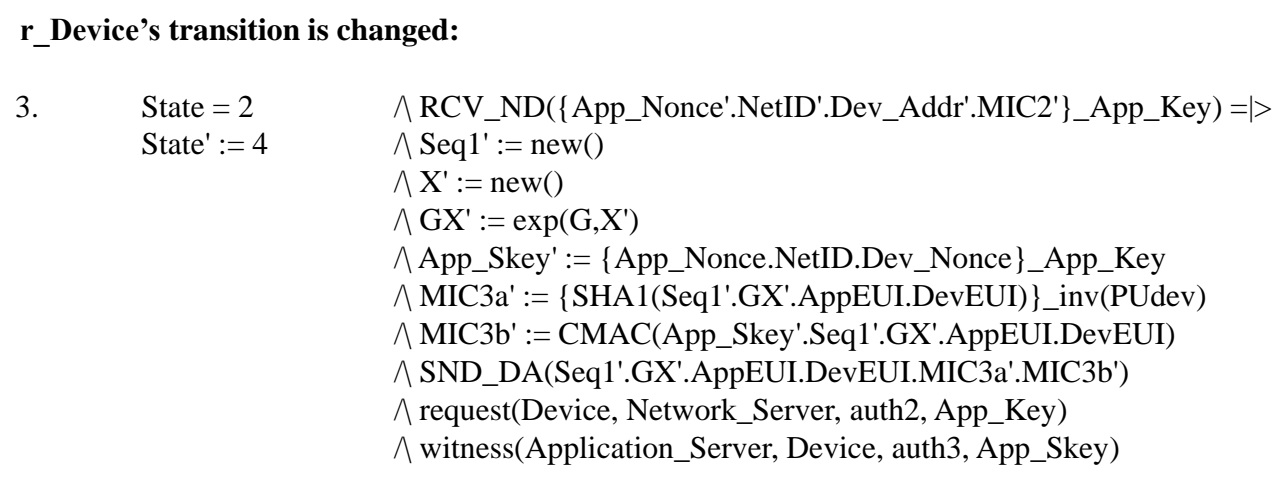

r_Application's transition is changed:

5. State $=3 \wedge$ RCV_DA(Seq1'.GX'.AppEUI'.DevEUI'.\{SHA1(Seq1'.GX'.AppEUI'.DevEUI') $\}_{-}$inv(PUdev) CMAC(App_Skey.Seq1'.GX'.AppEUI'.DevEUI')) =|>

State $^{\prime}:=5 \quad \wedge$ Seq2 $:=\operatorname{ADD}($ Seq1.1)

$\wedge \mathrm{Y}^{\prime}:=\operatorname{new}()$

$\wedge \mathrm{GY}^{\prime}:=\exp \left(\mathrm{G}, \mathrm{Y}^{\prime}\right)$

$\wedge \mathrm{GXY}^{\prime}:=\exp \left(\mathrm{GX}, \mathrm{Y}^{\prime}\right)$

$\wedge \mathrm{SK}^{\prime}:=$ SHA1 $(\mathrm{GXY} \cdot$.Seq2')

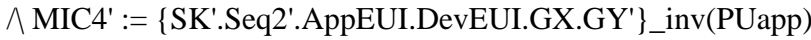

$\wedge$ MIC5' := CMAC(App_Skey.Seq2'.GY'.MIC4')

$\wedge$ SND_AD(Seq2'.GY'.MIC4'.MIC5')

$\wedge$ request(Device, Application_Server, auth3,App_Skey)

$\wedge \operatorname{secret}\left(S_{K}^{\prime}, \sec 2,\{\right.$ Application_Server, Device\})

Figure 12. Major changes in the Security-Enhanced Option (SEO).

Figures 13 and 14 illustrate the communication scenario between a client and a server for DTLS-PSK and DTLS-ECC, respectively. Both DTLS protocols are evaluated using the TinyDTLS 0.8.2 library with their respective cipher suite over the system with configurations presented in Table 4 .

Client_Hello (72 byte)

Figure 13. DTLS-ECC. 


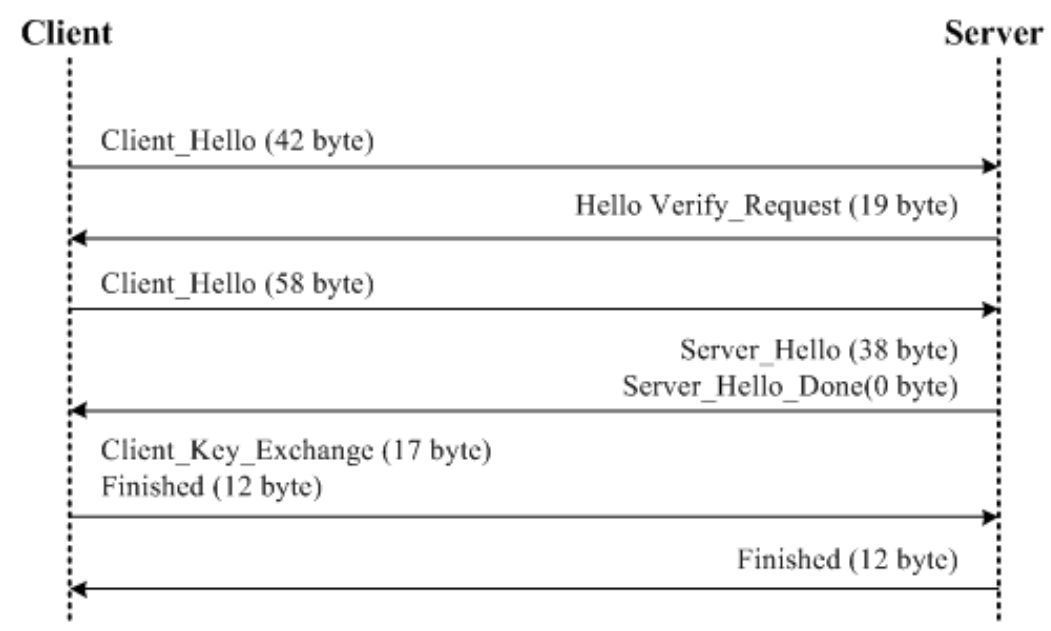

Figure 14. DTLS-PSK.

Table 4. Tiny DTLS-PSK 0.8.2 and Tiny DTLS-ECC 0.8.2 environments for evaluation.

\begin{tabular}{ll}
\hline Environment & Specific \\
\hline CPU & Intel $^{\circledR}$ Core $^{\mathrm{TM}}$ i5-6300HQ 2.30 GHz (0.88 GHz) \\
RAM & 8GB \\
Compiler & gcc (GCC) 6.4.0 \\
OS & Windows 10 64bit (Cygwin32) \\
DTLS Library & TinyDTLS 0.8.2 \\
Cipher Suite & TLS_ECDHE_ECDSA_WITH_AES_128_CCM_8 \\
Cipher Suite & TLS_PSK_WITH_AES_128_CCM_8 \\
\hline
\end{tabular}

DTLS-PSK uses client and server initiations along with the exchange messages followed by the finish mechanisms for handshake during a contact between the client and the server. The client messages for DTLS-PSK cause an overhead of $1 \mathrm{~ms}$ for each operation, whereas there is no overhead due to server-initiation. Here, overheads are measured as the difference between the time after sending a handshake message and the time utilized before generating a handshake message. The server-overhead is only caused by finishing the communication. This lesser dependence on the server causes fewer overheads on the communication setup, but with compromised security. The details of packet size and overheads for DTLS-PSK are provided in Table 5. Using each packet size, the total message size used for handshaking between the client and the server is calculated, as shown in Table 6. From these system-based evaluations, it is obtained that DTLS-PSK causes a total overhead of $5 \mathrm{~ms}$ with a total message size of 198 bytes.

Table 5. DTLS-PSK packet size and overheads.

\begin{tabular}{lll}
\hline Message & Size (Bytes) & Overhead (ms) \\
\hline Client_Hello(1) & 42 & 1 \\
Hello_Verify_Request & 19 & - \\
Client_Hello(2) & 58 & 1 \\
Server_Hello & 38 & - \\
Server_Hello_Done & 0 & - \\
Client_Key_Exchange & 17 & 1 \\
Finished(Client) & 12 & 1 \\
Finished(Server)_Verify & 12 & 1 \\
\hline Total Overhead & & 5 \\
\hline
\end{tabular}


Table 6. DTLS-PSK handshake message packet.

\begin{tabular}{ll}
\hline Message & Size (Bytes) \\
\hline Client_Hello(1) & 42 \\
Hello_Verify_Request & 19 \\
Client_Hello(2) & 58 \\
Server_Hello, Server Hello Done & 38 \\
Client_Key_Exchange, Finished & 29 \\
Finished & 12 \\
\hline Total Message Size & 198 \\
\hline
\end{tabular}

DTLS-ECC uses similar policies for a handshake as used by DTLS-PSK, but with the involvement of certification procedures for enhanced security of communication between the client and the server. The overhead scenarios are also similar to DTLS-PSK, i.e., $1 \mathrm{~ms}$, but excessive overheads are caused due to the certificate exchange and verification procedures that raise the overhead to $31 \mathrm{~ms}$. The details of total overhead for various procedures involved in the communication between the client and the server by using DTLS-ECC are presented in Table 7. Because of the certification scheme, DTLS-ECC uses a heavy packet size for a handshake between the involved entities. The maximum size packet is used for certificate-exchange procedures, which is 304 bytes, and the total message size reaches up to 746 bytes, as shown in Table 8 .

Table 7. DTLS-ECC packet size and overheads.

\begin{tabular}{lll}
\hline Message & Size (Bytes) & Overhead (ms) \\
\hline Client_Hello(1) & 72 & 1 \\
Hello_Verify_Request & 19 & - \\
Client Hello(2) & 88 & 1 \\
Server_Hello & 56 & - \\
Certificate & 97 & 1 \\
Server_Key_Exchange & 143 & - \\
Certificate_Request & 8 & - \\
Server_Hello_Done & 0 & - \\
Client_Key_Exchange & 66 & 31 \\
Certificate_Verify & 76 & 31 \\
Finished(Client) & 12 & 31 \\
Finished(Server) & 12 & 1 (for verification) \\
\hline Total Overhead & & 97 \\
\hline
\end{tabular}

Table 8. DTLS-ECC handshake message packet.

\begin{tabular}{ll}
\hline Message & Size (Bytes) \\
\hline Client_Hello(1) & 72 \\
Hello_Verify_Request & 19 \\
Client_Hello(2) & 88 \\
Server_Hello, Certificate, Server_Key_Exchange, Certificate_Request, Server_Hello_Done & 304 \\
Certificate, Client_Key_Exchange, Certificate_Verify, Finished & 251 \\
Finished & 12 \\
\hline Total Message Size & 746 \\
\hline
\end{tabular}

Similarly to DTLS-PSK and DTLS-ECC, it is required to calculate the system-based message size and overhead caused by the proposed protocol for its implementation in practical scenarios. The environment setup for system-based evaluations of the proposed protocol is presented in Table 9 . The proposed extended LoRaWAN protocol is evaluated for both its options. In DO, the overhead due to request and acknowledgment is $1 \mathrm{~ms}$, and that of response is $9 \mathrm{~ms}$. The message size varies between 37 and 53 bytes for request and response, respectively. The acknowledgment uses 4 bytes for its procedures. Thus, the total message size and the overhead for the proposed protocol in 
DO are 94 bytes and $11 \mathrm{~ms}$, respectively, as shown in Table 10. In SEO of the proposed protocol, a malicious network server is blocked from manipulating packets between a device and its application server along with impersonating them. Thus, SEO consumes more time in generating final responses. Overall, the mechanism is not much different, but the extra features increase the message size of the SEO approach. The system-based evaluation for the proposed approach in SEO suggests that the total message size reaches up to 126 bytes, and the total overhead is $16 \mathrm{~ms}$, as shown in Table 11 . A comparison is drawn in Table 12 to understand the impact on the system-based evaluation for DTLS-PSK, DTLS-ECC and extended LoRaWAN protocol with DO and SEO.

Table 9. Proposed protocol environment for evaluation.

\begin{tabular}{ll}
\hline Environment & Specific \\
\hline CPU & Intel $^{\circledR}$ Core $^{\mathrm{TM}}{ }_{\mathrm{i} 5}-6300 \mathrm{HQ} 2.30 \mathrm{GHz}(0.88 \mathrm{GHz})$ \\
RAM & $8 \mathrm{~GB}$ \\
Compiler & Visual Studio 2017 32 bit \\
OS & Windows 1064 bit \\
\hline
\end{tabular}

Table 10. Message size and overhead for the proposed protocol's Default Option (DO).

\begin{tabular}{lll}
\hline Message & Size (Bytes) & Overhead (ms) \\
\hline App_Auth_Req & 37 & 1 \\
APP_Auth_Res & 53 & 9 \\
App_Auth_ACK & 4 & 1 \\
\hline Total Overhead & & 11 \\
\hline Total Message Size & 94 & \\
\hline
\end{tabular}

Table 11. Message size and overhead for the proposed protocol's SEO.

\begin{tabular}{lll}
\hline Message & Size (Bytes) & Overhead (ms) \\
\hline App_Auth_Req & 69 & 3 \\
APP_Auth_Res & 53 & 12 \\
App_Auth_ACK & 4 & 1 \\
\hline Total Overhead & & 16 \\
\hline Total Message Size & 126 & \\
\hline
\end{tabular}

Table 12. System-based comparison for DTLS-PSK, DTLS-ECC and extended LoRaWAN protocol with DO and SEO features.

\begin{tabular}{lllllll}
\hline Protocol & Overhead (ms) & $\begin{array}{l}\text { Overhead } \\
\text { Impact } \\
\text { w.r.t. DO }\end{array}$ & $\begin{array}{l}\text { Overhead } \\
\text { Impact } \\
\text { w.r.t. SEO }\end{array}$ & Message Size (Bytes) & $\begin{array}{l}\text { Message } \\
\text { Size Impact } \\
\text { w.r.t. DO }\end{array}$ & $\begin{array}{l}\text { Message } \\
\text { Size Impact } \\
\text { w.r.t. SEO }\end{array}$ \\
\hline DO & 11 & - & $-31.25 \%$ & 94 & - & $-25.39 \%$ \\
SEO & 16 & $+31.25 \%$ & - & 126 & $+25.39 \%$ & - \\
DTLS-PSK & 5 & $-54.54 \%$ & $-68.75 \%$ & 198 & $+52.52 \%$ & $+36.36 \%$ \\
DTLS-ECC & 97 & $+88.65 \%$ & $+83.50 \%$ & 746 & $+87.39 \%$ & $+83.10 \%$ \\
\hline
\end{tabular}

\subsection{Evaluation of the Proposed Protocol over a Low-Power Device}

The system-based evaluations in the previous subsection help to determine the performance of the proposed protocol in a generic environment. However, the devices involved as end users in a LoRaWAN setup are of low-power ratings, such as mobiles, that may cause excessive overheads because of a difference in the type of CPU. To understand this variation, the two options, DO and SEO, of the proposed protocols are tested on a mobile platform with configurations given in Table 13. 
From the configurations, it can be identified that the CPU is limited in processing compared with system-based evaluations. The two options of the proposed protocol are coded separately for the Android version, and the results are recorded for overheads. The evaluations suggest that the proposed protocol with DO causes overhead in the range between 18 and 22 ms with a variation of $18.18 \%$, whereas the proposed protocol with SEO causes overhead in the range between 26 and $33 \mathrm{~ms}$ with a variation of $21.21 \%$, as shown in Table 14 . The results for overhead are low even for a low-power device, which illustrates significant improvements in terms of security without affecting the performance.

Table 13. Proposed protocol environment for evaluation over a mobile device.

\begin{tabular}{ll}
\hline Environment & Specific \\
\hline Device Type & Mobile \\
Make & Lenovo \\
Chipset & Mediatek MT6753 \\
CPU & Octa-core 1.3 GHz Cortex-A53 \\
GPU & Mali-T720MP3 \\
Compiler & Visual Studio 2017 (Android Kit) \\
OS & Android 5.1.1 \\
\hline
\end{tabular}

Table 14. Overhead for the proposed protocol (DO and SEO) over a low-power device.

\begin{tabular}{ccccc}
\hline Message & DO $(\mathbf{m i n})(\mathbf{m s})$ & DO $(\mathbf{m a x})(\mathbf{m s})$ & SEO $(\mathbf{m i n})(\mathbf{m s})$ & SEO $(\max )(\mathbf{m s})$ \\
\hline App_Auth_Req & 12 & 12 & 20 & 20 \\
APP_Auth_Res & 5 & 9 & 5 & 12 \\
App_Auth_ACK & 1 & 1 & 1 & 1 \\
Total Overhead & 18 & 22 & 26 & 33 \\
\hline
\end{tabular}

\subsection{Smart Factory-Enabled Parking System with the Proposed Protocol}

Further, in order to understand the behavior of the proposed protocol, a smart parking scenario is considered, as shown in Figure 15. The scenario is comprised of multiple parking spaces each equipped with sensors, which transmit traffic to a local body via a gateway. The local body provides support for the LoRa network server, which is connected to the application server that is placed at the central authority. This case study can be matched with a special case, where a smart factory wants to track all the processes and the available parking places across the state. This information can be passed directly to the intended users via particular applications. On the contrary, the application servers can be replaced with direct communication with the users. However, in such a case, the authentication of the user is also to be considered, which may cause excessive overheads. Nevertheless, considering the scenario illustrated in this paper, the results are recorded for network latency and signaling overheads.

Irrespective of the communication, the traffic in the considered smart factory-enabled parking scenario passes through a series of wired, as well as wireless links. Thus, network latency is calculated by considering a single wired and a single wireless link in the network. Since the traffic remains the same, a similar message size, as computed previously, is used for evaluating the overall latency of the network. Thus, in this scenario, the network latency is calculated as [64]:

$$
\mathcal{N}_{\mathcal{L}}^{(O)}=\mathcal{T}_{d}+\left(\frac{\mathcal{M}}{\mathcal{F}}-1\right) \mathcal{I}+L 2+\left(\frac{\mathcal{M K}}{\mathcal{B}}+\mathcal{W}_{d}\right)
$$

where $\mathcal{T}_{d}$ is the one-frame transport delay, $\mathcal{M}$ is the message size, $\mathcal{F}$ is the frame size, $\mathcal{I}$ is the inter-frame time, $L 2$ is the link layer delay, $\mathcal{K}$ is the number of intermediate hops, $\mathcal{B}$ is the network bandwidth and $\mathcal{W}_{d}$ is the wired delay. 


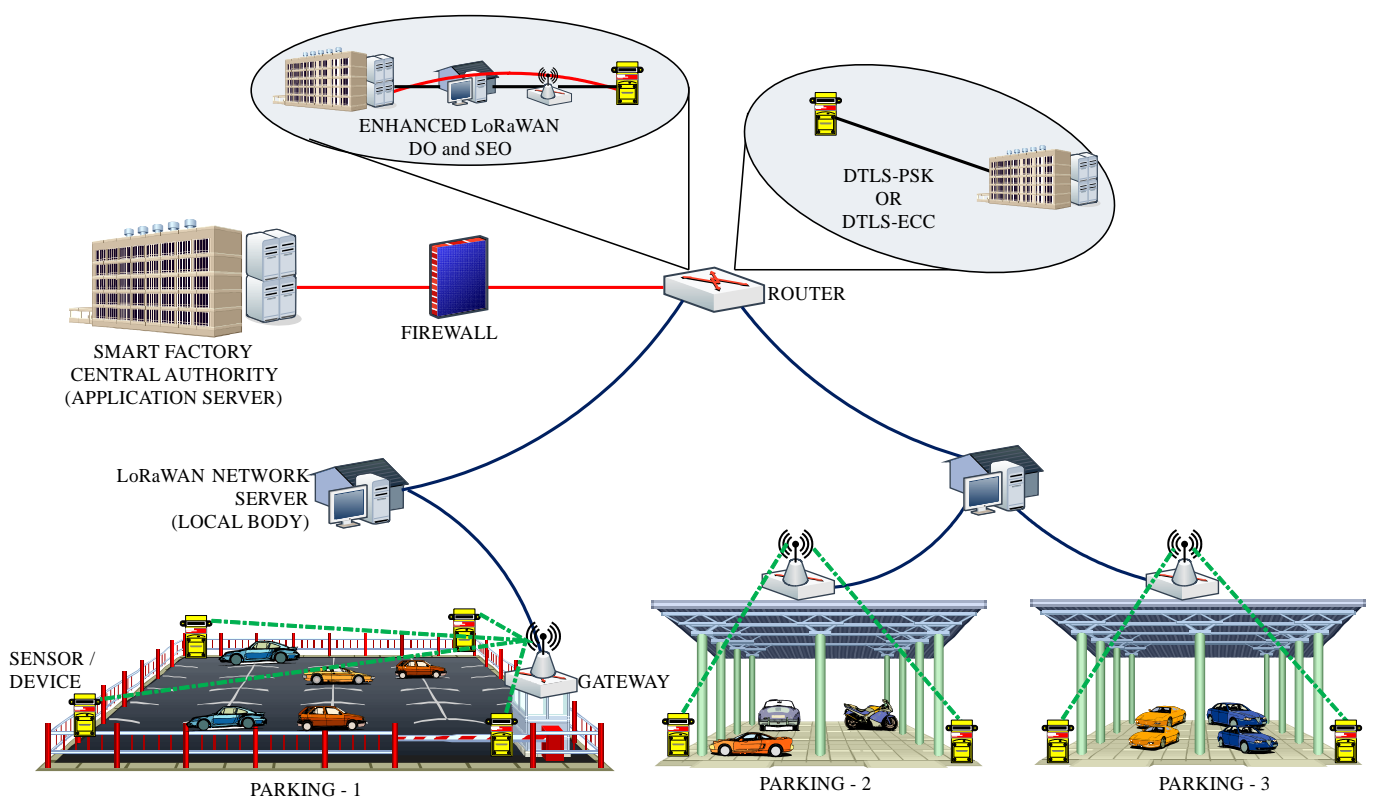

Figure 15. An illustration of the case study of protocols for a smart factory-enabled parking system.

Next, the signaling overheads are calculated for the proposed approach by following host-based principles as the devices or the sensors are responsible for initiating the communication in the proposed setup. Thus, signaling overhead is given as [65]:

$$
\mathcal{S}_{\mathcal{S}}^{O}=\frac{\mathcal{K} \mathcal{M}}{\tau},
$$

where $\tau$ is the session time between the involved entities. In case the network includes a master parking sensor, which manages all the sensors in the parking lot, the signaling overhead is calculated as:

$$
\mathcal{S}_{\mathcal{M}}^{O}=\frac{\mathcal{K} \mathcal{M}}{\tau}+\frac{(\mathcal{N}-1) \mathcal{K} \mathcal{M}}{\tau}
$$

where $\mathcal{N}$ is the number of sub-sensors in the parking lot. The details of values for each of the parameters used in the above formulations are given in Table 15.

Table 15. Parameter configurations.

\begin{tabular}{ll}
\hline Parameter & Value \\
\hline $\mathcal{N}$ & 10 \\
$\mathcal{B}$ & $11 \mathrm{Mbps}$ \\
$\mathcal{I}$ & $20 \mathrm{~ms}$ \\
$\mathcal{F}$ & 64 Bytes \\
$L 2$ & $45.35 \mathrm{~ms}$ \\
$\mathcal{T}_{d}$ & $10-50 \mathrm{~ms}$ \\
$\mathcal{K}$ & $1-10$ \\
$\tau$ & $100 \mathrm{~ms}$ \\
$\mathcal{W}_{d}$ & $20 \mathrm{~ms}$ \\
\hline
\end{tabular}

The proposed protocol operates with a maximum network latency of 485.4 and $485.5 \mathrm{~ms}$ for DO and SEO, respectively, whereas DTLS-PSK and DTLS-ECC operate with a maximum latency of 485.8 and $488.0 \mathrm{~ms}$, respectively, with variation in the number of intermediate hops. The results in Figure 16 show an enhanced performance of DO and SEO for the given smart parking scenario. The results for network latency are evaluated over Weibull fitting for understanding the reliability of the proposed enhanced LoRaWAN protocol in comparison with the DTLS-PSK and DTLS-ECC through MATLAB ${ }^{\mathrm{TM}}$, 
as shown in Figure 17. The reliability curves suggest that the proposed protocol performs better and offers a higher reliability of communication for the smart factory-enabled parking system. These results are dominated by the message size, and the overhead study presented in earlier parts plays a crucial role in deciding these outputs.

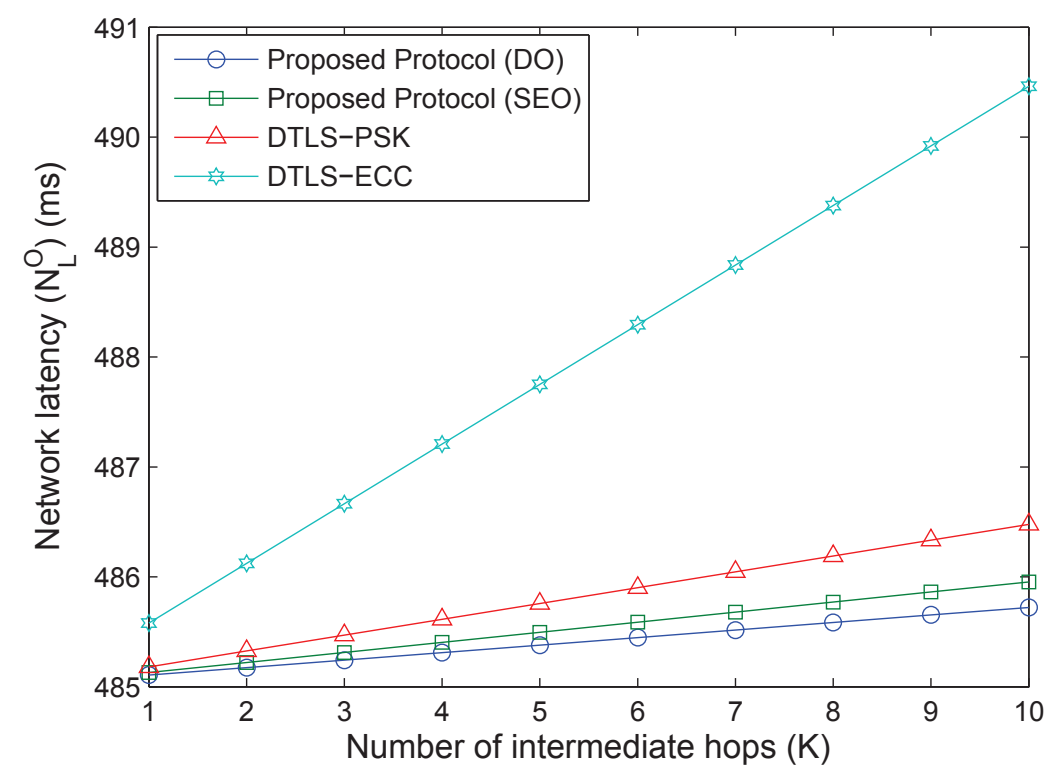

Figure 16. Network latency (ms) vs. the number of intermediate hops.

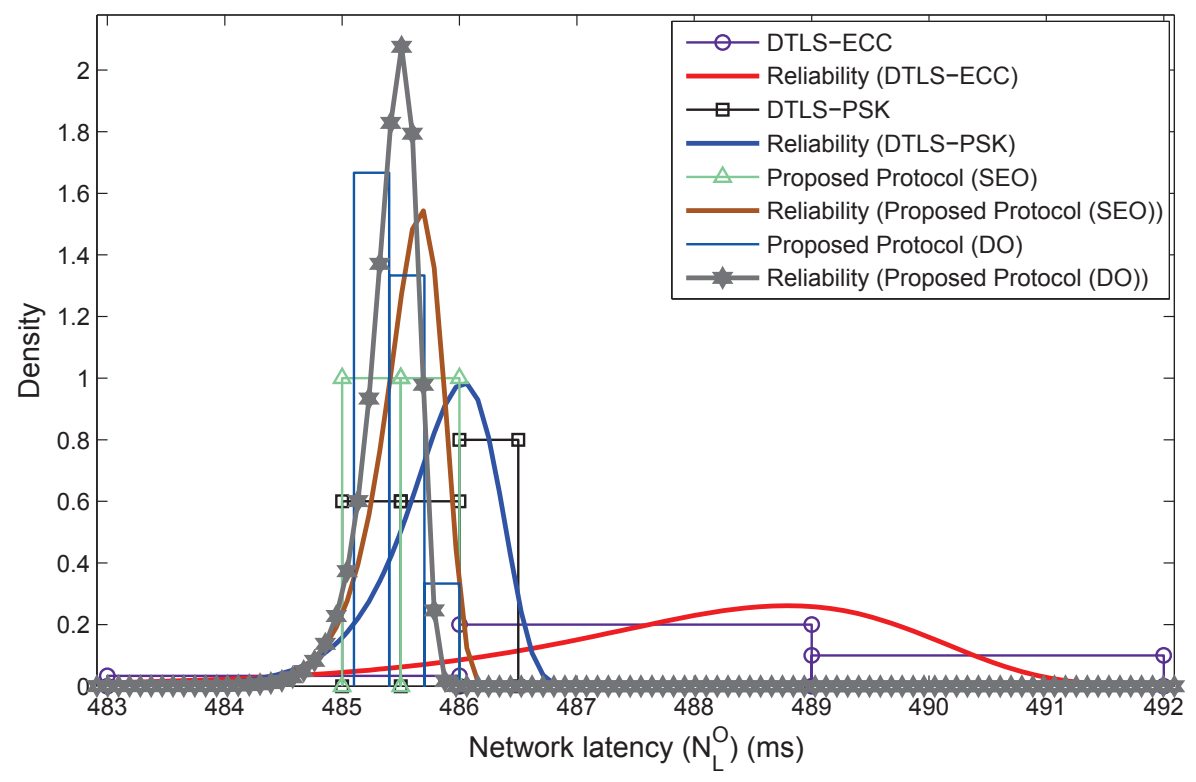

Figure 17. Density (network latency) vs. network latency with reliability fitting.

Network latency is also affected by the frame-delays over the wired links. This can be seen in Figure 18, which shows an increasing trend for all the protocols with an increase in the one-frame transport delay. Further, DTLS-ECC suffers from the highest latency because of the certification procedures that result in the higher message size. The average network latency for the proposed protocol with DO, the proposed protocol with SEO, DTLS-PSK and DTLS-ECC is 505.7, 505.9, 506.4 and $510.4 \mathrm{~ms}$, respectively. 

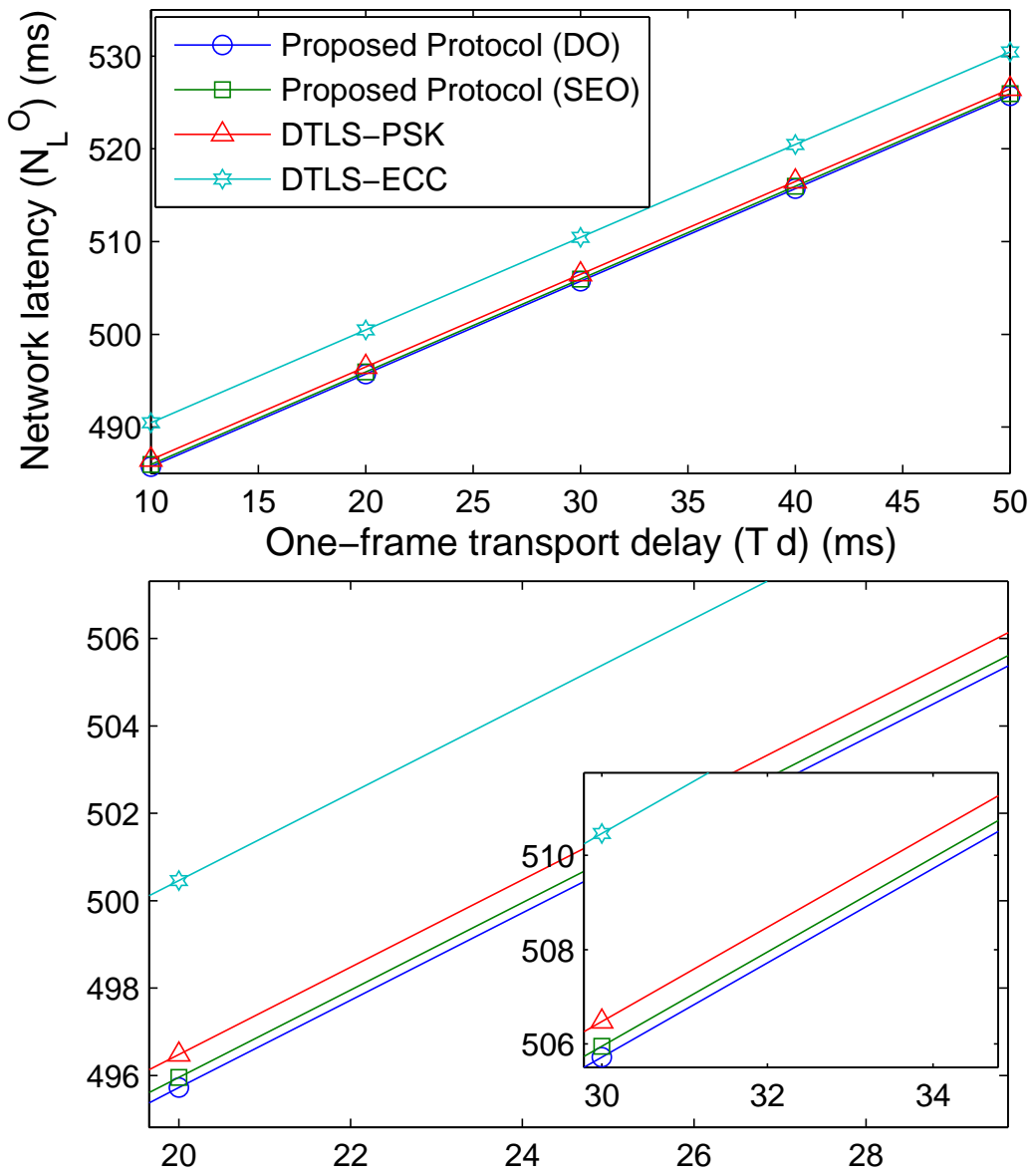

Figure 18. Network latency (ms) vs. transport delay.

Finally, results are recorded for signaling overheads by following (2) and (3), as shown in Figure 19. These results are recorded for two sub-scenarios in the considered smart parking case study. Firstly, the results include signaling overheads $\left(\mathcal{S}_{\mathcal{S}}^{O}\right)$ by using one-to-one communication between the application server and the single sensor, and secondly, the results include signaling overheads $\left(\mathcal{S}_{\mathcal{M}}^{O}\right)$ by using one-to-one communication between the application server and the single master sensor, which facilitates multiple sensors of a particular parking lot. The results show that the proposed protocol in DO causes $52.5 \%$ and $87.3 \%$ fewer signaling overheads than DTLS-PSK and DTLS-ECC, respectively; and the proposed protocol in SEO causes $36.3 \%$ and $83.1 \%$ fewer signaling overheads than DTLS-PSK and DTLS-ECC, respectively.

\subsection{Applicability to the New LoRaWAN Specification v1.1}

Recently, a new LoRaWAN specification v1.1, as shown in Figure 20, has been released, which covers the majority of the shortcomings of the LoRaWAN specification v1.02. The new specification comprises similar components as that of the v1.02 specification except that network server's functionalities are divided into home, serving and forwarding entities with security dependence on the join server. In the LoRaWAN specification v1.1, the data transferred between the application server and the end device are encrypted using the keys generated by the join server. Here, the involved network server aggregation is considered as trusted; however, a malicious network server may be able to alter the content of the data messages in transit, which may even help the compromised network server to infer some information about the data by observing the reaction of the application end-points to the altered data. Therefore, end-to-end security is needed between the application server and the 
end device, through which the data transmission can be protected in terms of confidentiality and integrity, and not compromised by the network server or any other entity.

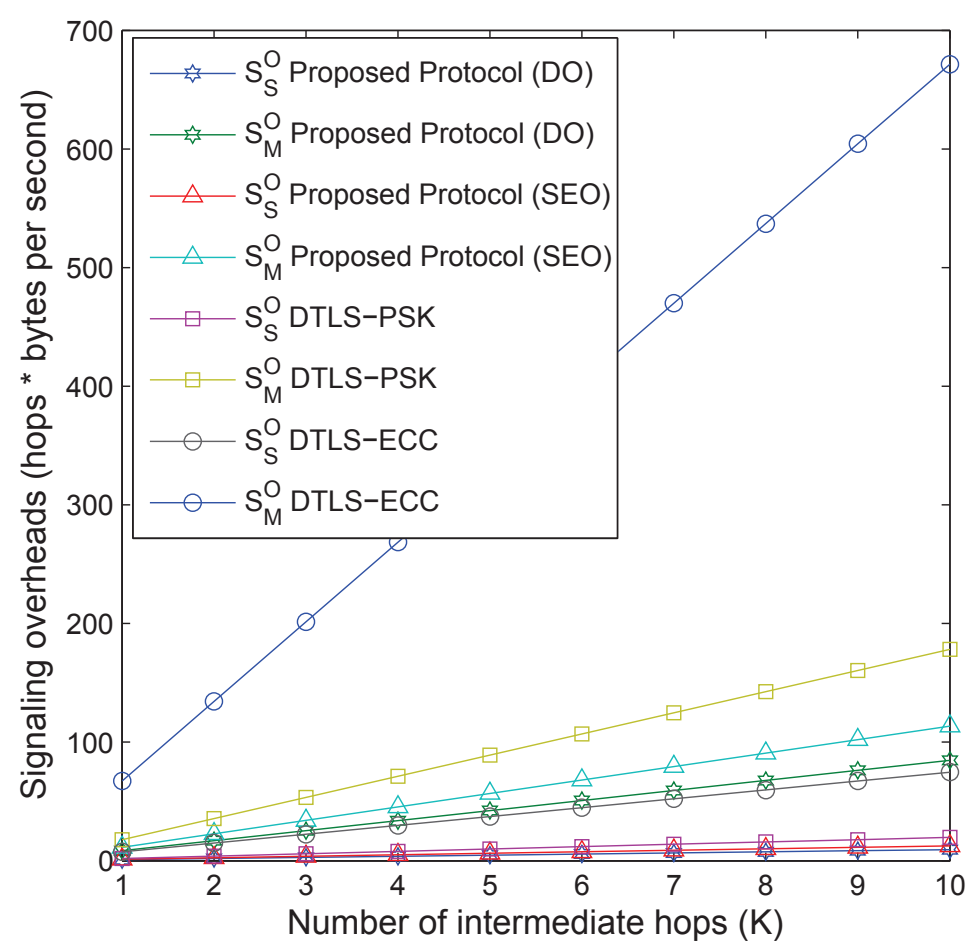

Figure 19. Signaling overheads vs. number of intermediate hops.

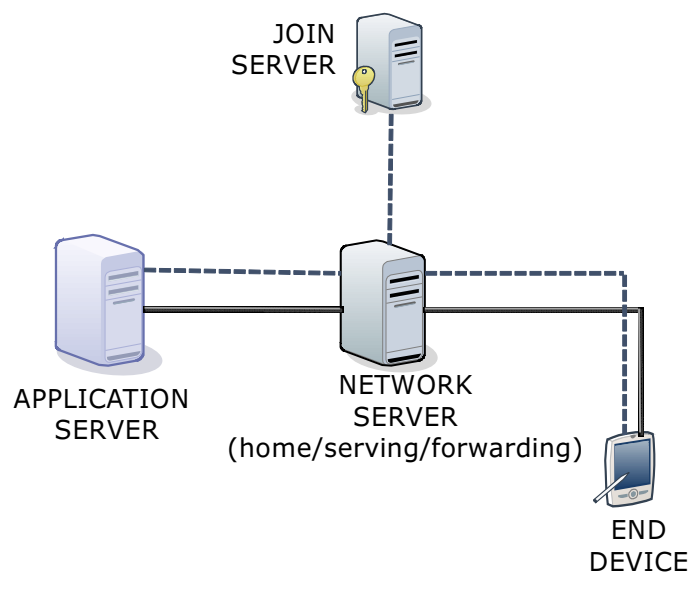

Figure 20. A general overview of the LoRaWAN specification v1.1 architecture.

These issues are further highlighted in the official release of the LoRa (https://www.loraalliance.org/lorawan-for-developers), Alliance ${ }^{\mathrm{TM}}$, as well as by the organizations (http://iotdesign. embedded-computing.com/articles/lora-networks-in-buildings-reduce-infrastructure-costs/; https: / / www.businesswire.com/news/home/20180220005492/en/Cypress-ESCRYPT-Unveil-End-to-endLoRaWAN-based-Security-Solution; https://micromaxtechnology.com/wp-content/uploads/GemaltoIoT-LoRaWAN-Brochure.pdf; https:/ /www.escrypt.com/en/solutions/secure-lorawan-communications) working on LoRaWAN applications. These detailed reports have clarified that the new specification assumes trust for network servers, but true end-to-end confidentiality and integrity protection are not yet covered by this new specification. Effective and secure strategic solutions are required for 
supporting the applications that have such requirements as their primary concern. All of these are supported by the proposed protocol that can secure the communications for both the LoRaWAN specifications v1.02 and v1.1.

It is to be noted that in the proposed protocol, DO focuses on preventing the passive attacks by the malicious network server, and SEO focuses on the active attacks by the malicious network server. Therefore, depending on the security requirements for the target service, a proper option can be selected for the LoRaWAN networks. The analyses presented in this paper by using BAN logic, the AVISPA tool, system simulations and the smart-parking case study demonstrate the performance of the proposed enhanced LoRaWAN protocol with the capability of securing the end-to-end communication between the device and the application server.

\section{Conclusions and Future Remarks}

Urban networks are largely affected by the need for smart applications, such as healthcare, city management, smart transportation and smart industry. Such networks are in demand, and with IoT, their applications can easily be extended across cities in the form of smart projects. In recent years, smart applications in the Internet of Things (IoT) were applied through a low-power communication setup, as the majority of devices in these networks are battery operated and smart applications running on them consume most of the battery life. One such facility is provided by the Low-Power Wide Area Network (LPWAN), but at a constrained bit rate. For facilitating long-range communication over LPWAN, several approaches and protocols are provided by different researchers and research organizations across the globe.

One of the popular protocols is the Long Range Wide Area Network (LoRaWAN), which is a media access layer protocol for long-range communication between the devices and the application servers via LPWAN gateways. However, LoRaWAN comes with issues related to security as a much-secured protocol will consume the majority of the resources (battery life) because of the excessive computational overheads and signaling cost. This may lead to several types of attacks on the network. The standard protocol fails to support the perfect forward secrecy, the end-to-end security and the defense against the replay attack. Thus, considering this as a problem, an enhanced LoRaWAN security protocol was proposed in this paper, which not only provides the functionalities of the basic protocol, but also prevents against different security threats.

The proposed protocol was developed with two options, the Default Option (DO) and the Security-Enhanced Option (SEO), to prevent a malicious network server from breaking the end-to-end security between a device and its application server. The initial security validations were conducted through the Burrows-Abadi-Needham (BAN) logic and the Automated Validation of Internet Security Protocols and Applications (AVISPA) tool. Next, system-based and low-power device-based performances were evaluated to understand the message size and the overhead of the proposed protocol. Further, for practical applications, the problem of a smart factory-enabled parking system was considered for secure and efficient parking management in smart cities. The results, in terms of network latency with reliability fitting and signaling overheads, show significant improvements and better performance for the proposed protocol in comparison with other security protocols, namely Datagram Transport Layer Security-Pre-Shared Key (DTLS-PSK) and Datagram Transport Layer Security-Elliptic Curve Cryptography (DTLS-ECC).

It is to be noted that the new LoRaWAN specification v1.1 is unable to support the end-to-end security between the user and the application server. This shortcoming can be overcome through our protocol, but details on the hardware-based implementation of the proposed protocol for LoRaWAN specification v1.1 will be presented in our future reports. 
Author Contributions: Conceptualization: I.Y., S.K., G.C., V.S. Formal analysis: I.Y., S.K. Funding acquisition: I.Y. Investigation: I.Y., G.C., V.S., J.T.S. Methodology: I.Y., G.C., V.S. Performance evaluation: G.C., S.K., V.S. Case study: V.S. Supervision: I.Y., V.S. Validation: I.Y., S.K., G.C., V.S., J.T.S. Visualization: I.Y., V.S. Writing, original draft: I.Y., V.S., G.C., S.K., J.T.S. Writing, review and editing: I.Y., V.S., J.T.S.

Funding: This work was supported by 'The Cross-Ministry Giga KOREA Project' grant funded by the Korea government (MSIT) (GK18N0300, Development of Indoor DAS Technology based on IFoF for 5G Mobile Communication) as well as the Soonchunhyang University Research Fund.

Conflicts of Interest: The authors declare no conflict of interest.

\section{References}

1. Hakiri, A.; Berthou, P.; Gokhale, A.; Abdellatif, S. Publish/subscribe-enabled software defined networking for efficient and scalable IoT communications. IEEE Commun. Mag. 2015, 53, 48-54. [CrossRef]

2. Saxena, N.; Roy, A.; Sahu, B.J.R.; Kim, H. Efficient IoT Gateway over 5G Wireless: A New Design with Prototype and Implementation Results. IEEE Commun. Mag. 2017, 55, 97-105. [CrossRef]

3. Sharma, V.; Kumar, R.; Kaur, R. UAV-assisted content-based sensor search in IoTs. Electron. Lett. 2017, 53, 724-726. [CrossRef]

4. Jayaraman, P.P.; Yavari, A.; Georgakopoulos, D.; Morshed, A.; Zaslavsky, A. Internet of things platform for smart farming: Experiences and lessons learnt. Sensors 2016, 16, 1884. [CrossRef] [PubMed]

5. Minoli, D.; Sohraby, K.; Occhiogrosso, B. IoT Considerations, Requirements, and Architectures for Smart Buildings x2014; Energy Optimization and Next-Generation Building Management Systems. IEEE Internet Things J. 2017, 4, 269-283.

6. Zhang, X.; Zhang, J.; Li, L.; Zhang, Y.; Yang, G. Monitoring citrus soil moisture and nutrients using an iot based system. Sensors 2017, 17, 447. [CrossRef] [PubMed]

7. Sun, X.; Ansari, N. Traffic Load Balancing among Brokers at the IoT Application Layer. IEEE Trans. Netw. Serv. Manag. 2017, doi:10.1109/TNSM.2017.2787859. [CrossRef]

8. Pratama, A.Y.N.; Zainudin, A.; Yuliana, M. Implementation of IoT-based passengers monitoring for smart school application. In Proceedings of the 2017 International Electronics Symposium on Engineering Technology and Applications (IES-ETA), Surabaya, Indonesia, 26-27 September 2017; pp. 33-38.

9. Li, G.; Zhou, H.; Li, G.; Feng, B. Application-aware and Dynamic Security Function Chaining for Mobile Networks. J. Internet Serv. Inf. Secur. 2017, 7, 21-34.

10. Sharma, V.; You, I.; Leu, F.; Atiquzzaman, M. Secure and efficient protocol for fast handover in $5 \mathrm{G}$ mobile Xhaul networks. J. Netw. Comput. Appl. 2018, 102, 38-57. [CrossRef]

11. Banerjee, D.; Dong, B.; Taghizadeh, M.; Biswas, S. Privacy-Preserving Channel Access for Internet of Things. IEEE Internet Things J. 2014, 1, 430-445. [CrossRef]

12. Baiardi, F.; Tonelli, F.; Isoni, L. Application Vulnerabilities in Risk Assessment and Management. J. Wirel. Mob. Netw. Ubiquitous Comput. Dependable Appl. 2016, 7, 41-59.

13. Wei, Z.; Zhao, B. A Space Information Service Forwarding Mechnism Based on Software Defined Network. J. Internet Serv. Inf. Secur. 2017, 7, 48-60.

14. Verma, S.; Kawamoto, Y.; Fadlullah, Z.M.; Nishiyama, H.; Kato, N. A Survey on Network Methodologies for Real-Time Analytics of Massive IoT Data and Open Research Issues. IEEE Commun. Surv. Tutor. 2017, 19, 1457-1477. [CrossRef]

15. Balid, W.; Refai, H.H. On the development of self-powered iot sensor for real-time traffic monitoring in smart cities. In Proceedings of the 2017 IEEE SENSORS, Glasgow, UK, 29 October-1 November 2017; pp. 1-3.

16. Fortino, G.; Russo, W.; Savaglio, C.; Shen, W.; Zhou, M. Agent-Oriented Cooperative Smart Objects: From IoT System Design to Implementation. IEEE Trans. Syst. Man Cybern. Syst. 2017, PP, 1-18, doi:10.1109/TSMC.2017.2780618. [CrossRef]

17. Sharma, V.; You, I.; Kumar, R. Resource-based mobility management for video users in 5G using catalytic computing. Comput. Commun. 2007, doi:10.1016/j.comcom.2017.10.009. [CrossRef]

18. Parada, R.; Melia-Segui, J. Gesture Detection Using Passive RFID Tags to Enable People-Centric IoT Applications. IEEE Commun. Mag. 2017, 55, 56-61. [CrossRef]

19. Li, C.; Peng, Z.; Huang, T.Y.; Fan, T.; Wang, F.K.; Horng, T.S.; Muñoz-Ferreras, J.M.; Gómez-García, R.; Ran, L.; Lin, J. A review on recent progress of portable short-range noncontact microwave radar systems. IEEE Trans. Microwave Theory Tech. 2017, 65, 1692-1706. [CrossRef] 
20. Kong, L.; Khan, M.K.; Wu, F.; Chen, G.; Zeng, P. Millimeter-wave wireless communications for IoT-cloud supported autonomous vehicles: Overview, design, and challenges. IEEE Commun. Mag. 2017, 55, 62-68. [CrossRef]

21. Singh, R.; Singh, E.; Nalwa, H.S. Inkjet printed nanomaterial based flexible radio frequency identification (RFID) tag sensors for the internet of nano things. RSC Adv. 2017, 7, 48597-48630. [CrossRef]

22. Wu, X.; Li, F. A multi-domain trust management model for supporting RFID applications of IoT. PLoS ONE 2017, 12, e0181124, doi:10.1371/journal.pone.0181124. [CrossRef] [PubMed]

23. Petäjäjärvi, J.; Mikhaylov, K.; Hämäläinen, M.; Iinatti, J. Evaluation of LoRa LPWAN technology for remote health and wellbeing monitoring. In Proceedings of the 2016 10th International Symposium on Medical Information and Communication Technology (ISMICT), Worcester, MA, USA, 20-23 March 2016; pp. 1-5.

24. Bardyn, J.P.; Melly, T.; Seller, O.; Sornin, N. IoT: The era of LPWAN is starting now. In Proceedings of the 2016: 42nd European Solid-State Circuits Conference, Lausanne, Switzerland, 12-15 September 2016; pp. 25-30.

25. Garcia-Carrillo, D.; Marin-Lopez, R.; Kandasamy, A.; Pelov, A. A CoAP-Based Network Access Authentication Service for Low-Power Wide Area Networks: LO-CoAP-EAP. Sensors 2017, 17, 2646, doi:10.3390/s17112646. [CrossRef] [PubMed]

26. Sanchez-Iborra, R.; Gamez, J.S.; Santa, J.; Fernandez, P.J.; Skarmeta, A.F. Integrating LP-WAN Communications within the Vehicular Ecosystem. J. Internet Serv. Inf. Secur. 2017, 7, 45-56.

27. Sánchez, B.B.; Alcarria, R.; de Rivera, D.S.; Sánchez-Picot, Á. Enhancing Process Control in Industry 4.0 Scenarios using Cyber-Physical Systems. JoWUA 2016, 7, 41-64.

28. Desnitsky, V.; Levshun, D.; Chechulin, A.; Kotenko, I.V. Design Technique for Secure Embedded Devices: Application for Creation of Integrated Cyber-Physical Security System. JoWUA 2016, 7, 60-80.

29. Carniani, E.; Costantino, G.; Marino, F.; Martinelli, F.; Mori, P. Enhancing Video Surveillance with Usage Control and Privacy-Preserving Solutions. JoWUA 2016, 7, 20-40.

30. Li, J.; Li, J.; Chen, X.; Jia, C.; Lou, W. Identity-based encryption with outsourced revocation in cloud computing. IEEE Trans. Comput. 2015, 64, 425-437. [CrossRef]

31. Li, P.; Li, J.; Huang, Z.; Li, T.; Gao, C.Z.; Yiu, S.M.; Chen, K. Multi-key privacy-preserving deep learning in cloud computing. Future Gener. Comput. Syst. 2017, 74, 76-85. [CrossRef]

32. Li, P.; Li, J.; Huang, Z.; Gao, C.Z.; Chen, W.B.; Chen, K. Privacy-preserving outsourced classification in cloud computing. Clust. Comput. 2017, doi:10.1007/s10586-017-0849-9. [CrossRef]

33. Li, J.; Zhang, Y.; Chen, X.; Xiang, Y. Secure attribute-based data sharing for resource-limited users in cloud computing. Comput. Secur. 2018, 72, 1-12. [CrossRef]

34. Huang, Z.; Liu, S.; Mao, X.; Chen, K.; Li, J. Insight of the Protection for Data Security under Selective Opening Attacks. Inf. Sci. 2017, doi:10.1016/j.ins.2017.05.031. [CrossRef]

35. Bankov, D.; Khorov, E.; Lyakhov, A. Mathematical model of LoRaWAN channel access. In Proceedings of the 2017 IEEE 18th International Symposium on a World of Wireless, Mobile and Multimedia Networks (WoWMoM), Macau, China, 12-15 June 2017; pp. 1-3.

36. Lanza, J.; Sánchez, L.; Muñoz, L.; Galache, J.A.; Sotres, P.; Santana, J.R.; Gutiérrez, V. Large-Scale Mobile Sensing Enabled Internet-of-Things Testbed for Smart City Services. Int. J. Distrib. Sens. Netw. 2015, 11, doi:10.1155/2015/785061. [CrossRef]

37. Park, S.; Park, S.; Byun, J.; Park, S. Design of a mass-customization-based cost-effective Internet of Things sensor system in smart building spaces. Int. J. Distrib. Sens. Netw. 2016, 12. [CrossRef]

38. Adelantado, F.; Vilajosana, X.; Tuset-Peiro, P.; Martinez, B.; Melia-Segui, J.; Watteyne, T. Understanding the limits of LoRaWAN. IEEE Commun. Mag. 2017, 55, 34-40. [CrossRef]

39. Zulian, S. Security Threat Analysis and Countermeasures for LoRaWAN Join Procedure, 2016. Available online: https:/ labs.mwrinfosecurity.com/publications/lo/ (accessed on 26 December 2017).

40. Miller, R. LoRa Security: Building a Secure LoRa Solution, 23 March 2016. Available online: https:/ /labs. mwrinfosecurity.com/publications/lo/ (accessed on 26 December 2017).

41. Na, S.; Hwang, D.; Shin, W.S.; Kim, K.H. Scenario and Countermeasure for Replay Attack Using Join Request Messages in LoRaWAN. In Proceedings of the 2017 International Conference on Information Networking (ICOIN), Da Ning, Vietnam, 11-13 January 2017.

42. Kim, J.; Song, J. A Dual Key-Based Activation Scheme for Secure LoRaWAN. Hindawi Wirel. Commun. Mob. Comput. 2017, 2017, 6590713. [CrossRef] 
43. Garcia, D.; Marin, R.; Kandasamy, A.; Pelov, A. LoRaWAN Authentication in Diameter Draft-Garcia-Dime-Diameter-Lorawan-00, 30 May 2016. Available online: https://tools.ietf.org/html/ draft-garcia-dime-diameter-lorawan-00 (accessed on 26 December 2017).

44. Garcia, D.; Marin, R.; Kandasamy, A.; Pelov, A. LoRaWAN Authentication in RADIUS Draft-Garcia-Radext-Radius-Lorawan-03, 2 May 2017. Available online: https:/ /www.ietf.org/archive/id/ draft-garcia-radext-radius-lorawan-03.txt (accessed on 26 December 2017).

45. Fossati, T.; Tschofenig, H. Transport Layer Security (TLS)/Datagram Transport Layer Security (DTLS) Profiles for the Internet of Things. IETF RFC 7925, 2016. Available online: https://tools.ietf.org/html/rfc7925 (accessed on 1 May 2018).

46. Burrows, M.; Abadi, M.; Needham, R. A logic of authentication. ACM Trans. Comput. Syst. 1990, 8, 18-36. [CrossRef]

47. Viganò, L. Automated Security Protocol Analysis with the AVISPA Tool. Electron. Notes Theor. Comput. Sci. 2006, 155, 61-86. [CrossRef]

48. Koulamas, C.; Giannoulis, S.; Fournaris, A. IoT Components for Secure Smart Building Environments. In Components and Services for IoT Platforms; Springer: New York, NY, USA, 2017; pp. 335-353.

49. Farrell, S. LPWAN Overview: Draft-ietf-lpwan-overview-01. Available online: https://tools.ietf.org/html/ draft-ietf-lpwan-overview-01 (accessed on 1 May 2018).

50. Casals, L.; Mir, B.; Vidal, R.; Gomez, C. Modeling the Energy Performance of LoRaWAN. Sensors 2017, 17, 2364, doi:10.3390/s17102364. [CrossRef] [PubMed]

51. Sornin, N.M.; Luis, T.E.T.K.; Hersent, O. LoRaWAN Specification V1.0.2. Available online: https://loraalliance.org/resource-hub/lorawantm-specification-v102 (accessed on 1 May 2018).

52. Girard, P. Low Power Wide Area Networks Security, 9 December 2015. Available online: https:/ / docbox. etsi.org/Workshop/2015/201512_M2MWORKSHOP/S04_WirelessTechnoforIoTandSecurityChallenges / GEMALTO_GIRARD.pdf (accessed on 26 December 2017).

53. Avoine, G.; Ferreira, L. Rescuing LoRaWAN 1.0. 2017. Available online: https://fc18.ifca.ai/preproceedings / 13.pdf (accessed on 15 May 2018).

54. Miller, V.S. Use of Elliptic Curves in Cryptography. In Advances in Cryptology-CRYPTO'85 Proceedings; Springer: Berlin/Heidelberg, Germany, 1985; Volume 218, pp. 417-426.

55. Johnson, D.; Menezes, A.; Vanstone, S. The Elliptic Curve Digital Signature Algorithm (ECDSA). Int. J. Inf. Secur. 2001, 1, 26-63. [CrossRef]

56. You, I.; Hori, Y.; Sakurai, K. Enhancing SVO Logic for Mobile IPv6 Security Protocols. J. Wirel. Mob. Netw. Ubiquitous Comput. Dependable Appl. 2011, 2, 26-52.

57. Shin, D.; Sharma, V.; Kim, J.; Kwon, S.; You, I. Secure and Efficient Protocol for Route Optimization in PMIPv6-Based Smart Home IoT Networks. IEEE Access 2017, 5, 11100-11117. [CrossRef]

58. Menezes, A.J.; van Oorschot, P.C.; Vanstone, S.A. Handbook of Applied Cryptography; CRC Press: Boca Raton, FL, USA, 1996.

59. Chevalier, Y.; Compagna, L.; Cuellar, J.; Drielsma, P.; Mantovani, J.; Mödersheim, S.; Vigneron, L. A High Level Protocol Specification Language for Industrial Security-Sensitive Protocols. In Proceedings of the Workshop on Specification and Automated Processing of Security Requirements, Linz, Austria, 20-24 September 2004.

60. Basin, D.; Mödersheim, S.; Viganò, L. OFMC: A symbolic model checker for security protocols. Electron. Notes Theor. Comput. Sci. 2005, 4, 61-86. [CrossRef]

61. Alferes, J.J.; Leite, J. SATMC: A SAT-Based Model Checker for Security Protocols. In European Workshop on Logics in Artificial Intelligence-JELIA 2004: Logics in Artificial Intelligence; Springer: Berlin/Heidelberg, Germany; Lisbon, Portugal, 2004; Volume 3229, pp. 730-733.

62. Turuani, M. SATMC: A SAT-Based Model Checker for Security Protocols. In International Conference on Rewriting Techniques and Applications_RTA 2006: Term Rewriting and Applications; Springer: Berlin/Heidelberg, Germany; Seattle, WA, USA, 2006; Volume 4098, pp. 277-286.

63. Boichut, Y.; Heam, P.C.; Kouchnarenko, O.; Oehl, F. Improvements on the Genet and Klay Technique to Automatically Verify Security Protocols. In Proceedings of the ETAPS 2004 Workshop on Automated Verification of Infinite States Systems (AVIS 2004), Barcelona, Spain, 3-4 April 2004; pp. 1-11. 
64. Guan, J.; Sharma, V.; You, I.; Atiquzzaman, M. Extension of MIH to Support FPMIPv6 for Optimized Heterogeneous Handover. arXiv 2017, arXiv:1705.09835. [CrossRef]

65. Lee, J.H.; Bonnin, J.M.; Seite, P.; Chan, H.A. Distributed IP mobility management from the perspective of the IETF: Motivations, requirements, approaches, comparison, and challenges. IEEE Wirel. Commun. 2013, 20, 159-168. [CrossRef]

(C) 2018 by the authors. Licensee MDPI, Basel, Switzerland. This article is an open access article distributed under the terms and conditions of the Creative Commons Attribution (CC BY) license (http:/ / creativecommons.org/licenses/by/4.0/). 